\title{
Mitigating the electron cloud effect in the SuperKEKB positron ring
}

\author{
Y. Suetsugu, H. Fukuma, K. Ohmi, M. Tobiyama, J. Flanagan, H. Ikeda, E. Mulyani, K. Shibata, \\ T. Ishibashi, M. Shirai, S. Terui, K. Kanazawa, and H. Hisamatsu \\ High Energy Accelerator Research Organization (KEK), 305-0801, Tsukuba, Japan
}

(Received 27 October 2018; published 1 February 2019)

\begin{abstract}
SuperKEKB is an electron-positron collider with asymmetric energies located at the High Energy Accelerator Research Organization (KEK) in Tsukuba, Japan. After more than five years of upgrading work on KEKB, phase-1 commissioning commenced in February 2016 and ended in June of the same year. Following a 20-month shutdown for the installation of a particle detector, BELLE II, phase-2 commissioning commenced in March 2018 and ended in July of the same year. This paper describes one major issue faced by SuperKEKB: the electron cloud effect (ECE) in the positron ring, which was observed during phase-1 commissioning. In the high-beam-current region, electron clouds, i.e., the source of the ECE, existed in the beam pipes at drift spaces of the ring, which had antechambers and a titanium nitride (TiN) film coating as countermeasures against the ECE. Permanent magnets and solenoids used to generate magnetic fields in the beam direction were attached to the beam pipes as additional countermeasures before the next commissioning phase commenced. Consequently, during phase- 2 commissioning, experiments showed that the threshold of the current linear density for exciting the ECE increased by a factor of at least 2 compared to that during phase- 1 commissioning. While the countermeasures were strengthened, the effectiveness of the antechambers and TiN film coating of the real beam pipe was reevaluated. Through various simulations and dedicated experiments during phase- 2 commissioning, the antechambers were found to be less effective than anticipated with regard to reducing the number of photoelectrons in the beam channel. In contrast, the TiN film coating had a low secondary electron yield, as expected.
\end{abstract}

DOI: 10.1103/PhysRevAccelBeams.22.023201

\section{INTRODUCTION}

SuperKEKB is an electron-positron collider with asymmetric energies located at the High Energy Accelerator Research Organization (KEK) in Tsukuba, Japan, and is a successor to the former KEKB (KEKB B-factory) [1-3]. The collider consists of an injector, a damping ring for positrons, a main ring (MR), and the Belle II particle detector (Fig. 1). The MR itself consists of two rings, each with a circumference of $3016 \mathrm{~m}$. The high- and low-energy rings (HER/LER) are for $7.0 \mathrm{GeV}$ electrons and $4.0 \mathrm{GeV}$ positrons, respectively. Each ring is composed of four arc sections and four straight sections, with lengths of approximately 550 and $200 \mathrm{~m}$, respectively. The straight sections include a beam injection/abort region, wiggler regions, radio-frequency accelerating cavity regions, and a beam collision region (Tsukuba section including Belle II detector). The design beam currents are $2.6 \mathrm{~A}$ (HER) and 3.6 A (LER), with a

\footnotetext{
*yusuke.suetsugu@kek.jp
}

Published by the American Physical Society under the terms of the Creative Commons Attribution 4.0 International license. Further distribution of this work must maintain attribution to the author(s) and the published article's title, journal citation, and DOI. maximum bunch number of 2500 and bunch spacing of two rf buckets (one rf bucket corresponds to approximately $2 \mathrm{~ns}$ in the case of SuperKEKB). The design

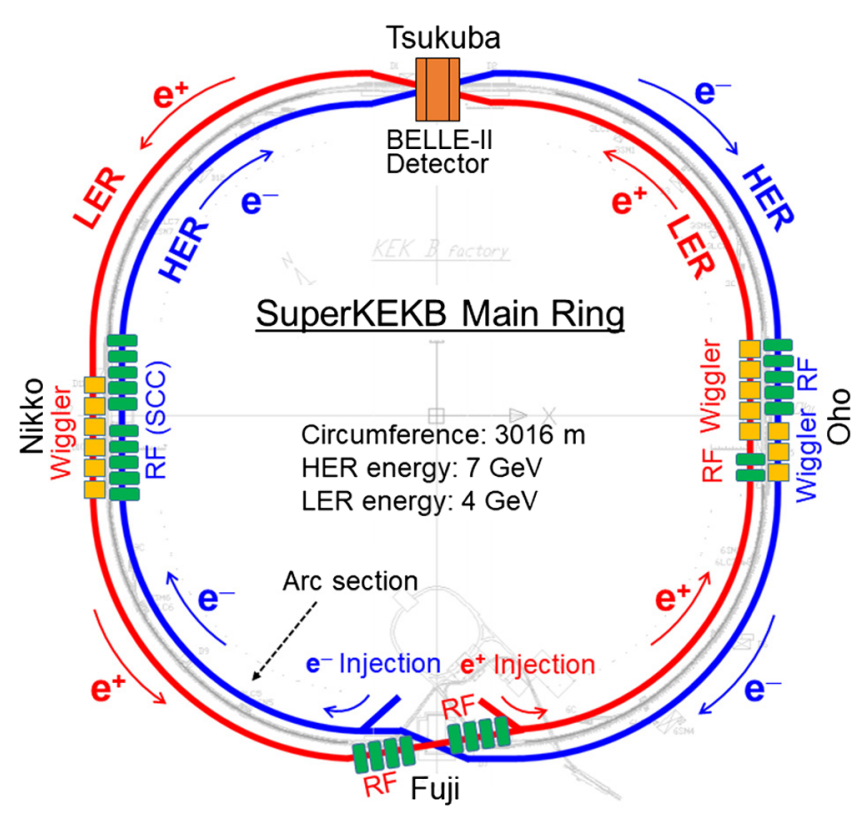

FIG. 1. Layout of the SuperKEKB main ring (MR). One ring consists of four arc sections and four straight sections. 
luminosity is $8 \times 10^{35} \mathrm{~cm}^{-2} \mathrm{~s}^{-1}$, which is approximately 40 times higher than that of KEKB [2].

The electron cloud effect (ECE) has been a serious problem in recent high-intensity proton and positron rings, including the SuperKEKB LER [4-18]. Note that here, the ECE signifies the effects on vacuum pressures as well as the beam size (emittance) of the positron beam. For example, the blowup of beam emittance caused by the ECE drastically affects the luminosity $[19,20]$. Simulations suggested that the threshold electron density $\left(n_{e_{\text {th }}}\left[\mathrm{m}^{-3}\right]\right)$ at which the blowup of the beam size occurs is estimated to be $\sim 3 \times$ $10^{11} \mathrm{~m}^{-3}[17,21]$. Hence, highly effective countermeasures against the ECE were required for the SuperKEKB LER [17,21-24]. Various ECE countermeasures were prepared on the basis of studies in numerous laboratories, such as CERN [10,11,25-31], Cornell University [15,32-34], SLAC [12,35-37], INFN [38,39], BNL [40,41], BINP [42,43], and of course, KEK [13,14,19,33,44-50].

After approximately five years of upgrading work starting in 2010, the first (phase-1) beam commissioning of SuperKEKB commenced in February 2016 and ended in June of the same year [51]. Phase-1 commissioning was dedicated to accelerator tuning. After a 20-month shutdown for the installation of the Belle II detector and the final focusing superconducting electromagnets, the second (phase-2) beam commissioning commenced in March 2018 and ended in July of the same year. Phase- 2 commissioning was dedicated to collision tuning and optics tuning for verifying the nanobeam scheme [1,2]. Various observations and studies of the ECE were performed during phase-1 and phase- 2 commissioning. During phase- 1 commissioning, the ECE was caused by the electron cloud in the beam pipes at drift spaces in the high-current-region, although these beam pipes had antechambers and a titanium-nitride
(TiN) film coating as countermeasures against ECE. After phase-1 commissioning, magnetic fields in the beam direction $\left(B_{z}[\mathrm{mT}]\right)$ were applied to the beam pipes as an additional countermeasure. As a result, the ECE was well suppressed and was not observed during phase- 2 commissioning. The countermeasures for SuperKEKB have been functioning well up to now. While the countermeasures were strengthened, the effectiveness of the antechambers and TiN film coating of the real beam pipes in the ring was reevaluated through various simulations and dedicated experiments during phase- 2 commissioning.

In the remainder of this paper, the results from various studies to mitigate the ECE during phase-1 and phase-2 commissioning are described. Furthermore, the results from the reevaluation of the effectiveness of the antechambers and TiN film coating for suppressing the ECE in the real beam pipe are reported.

\section{COUNTERMEASURES ADOPTED FOR SUPERKEKB}

The countermeasures adopted for the SuperKEKB LER are summarized in Table I [22-24].

Antechambers for suppressing the effect of photoelectrons and a TiN film coating for suppressing the secondary electron yield (SEY) $[25,26,33-37,40,41,44-46,52,53]$ were used in the majority of the new beam pipes, which were made of aluminum (Al) alloy. A schematic of a typical beam pipe at an arc section is presented in Fig. 2. The diameter of the beam channel is $90 \mathrm{~mm}$, the half aperture including the antechamber part is $110 \mathrm{~mm}$, and the height of the antechamber is $14 \mathrm{~mm}$. The TiN film has a typical thickness of $200 \mathrm{~nm}$ and was mainly coated on the beam channel. The beam pipes for bending magnets have longitudinal grooves in the beam

TABLE I. Countermeasures used to minimize the ECE in the SuperKEKB LER. The circles indicate the countermeasures applied for each main section in the ring $[22,24]$. Abbreviations: rf cavity section: beam pipes around rf cavities; IR: interaction region; $n_{e}$ (circular): electron density expected for circular beam pipe (copper); $n_{e}$ (expected): electron density expected after applying countermeasures; Antechamber: antechamber scheme; Solenoid: solenoid winding, manifested as a magnetic field in the beam direction $\left(B_{z}\right)$; Groove: beam pipe with grooves; Electrode: beam pipe with clearing electrodes.

\begin{tabular}{|c|c|c|c|c|c|c|c|c|}
\hline \multirow[b]{2}{*}{ Sectios } & \multicolumn{8}{|c|}{ Countermeasures } \\
\hline & $\begin{array}{l}\text { Length } \\
\text { [m] }\end{array}$ & $\begin{array}{c}n_{e} \text { (circular) } \\
{\left[\mathrm{m}^{-3}\right]}\end{array}$ & $\begin{array}{c}\text { Antechamber } \\
(1 / 5)\end{array}$ & $\begin{array}{c}\text { TiN coating } \\
(3 / 5)\end{array}$ & $\begin{array}{l}\text { Solenoid }\left(B_{z}\right) \\
\quad(1 / 50)\end{array}$ & $\begin{array}{c}\text { Groove } \\
(1 / 2)\end{array}$ & $\begin{array}{c}\text { Electrode } \\
(1 / 100)\end{array}$ & $\begin{array}{c}n_{e}(\text { expected }) \\
{\left[\mathrm{m}^{-3}\right]}\end{array}$ \\
\hline Drift space (arc) & 1629 & $8 \times 10^{12}$ & $\bigcirc$ & $\bigcirc$ & $\bigcirc$ & & & $2 \times 10^{10}$ \\
\hline Corrector magnet & 316 & $8 \times 10^{12}$ & $\bigcirc$ & $\bigcirc$ & $\bigcirc$ & & & $2 \times 10^{10}$ \\
\hline Bending magnet & 519 & $1 \times 10^{12}$ & $\bigcirc$ & $\bigcirc$ & & $\bigcirc$ & & $6 \times 10^{10}$ \\
\hline Wiggler magnet & 154 & $4 \times 10^{12}$ & 0 & $\bigcirc^{\mathrm{a}}$ & & & $\bigcirc$ & $5 \times 10^{9}$ \\
\hline $\begin{array}{l}\text { Quadrupole and sextupole } \\
\text { magnet }\end{array}$ & 254 & $4 \times 10^{10}$ & $\bigcirc$ & $\bigcirc$ & & & & $5 \times 10^{9}$ \\
\hline rf cavity section & 124 & $1 \times 10^{11}$ & & $\bigcirc$ & $\bigcirc$ & & & $1 \times 10^{9}$ \\
\hline IR & 20 & $5 \times 10^{11}$ & & $\bigcirc$ & $\bigcirc$ & & & $6 \times 10^{9}$ \\
\hline Total & 3016 & & & & & & & \\
\hline Average & & $5.5 \times 10^{12}$ & & & & & & $2.4 \times 10^{10}$ \\
\hline
\end{tabular}

${ }^{\mathrm{a}}$ Except for beam pipes with clearing electrodes. 


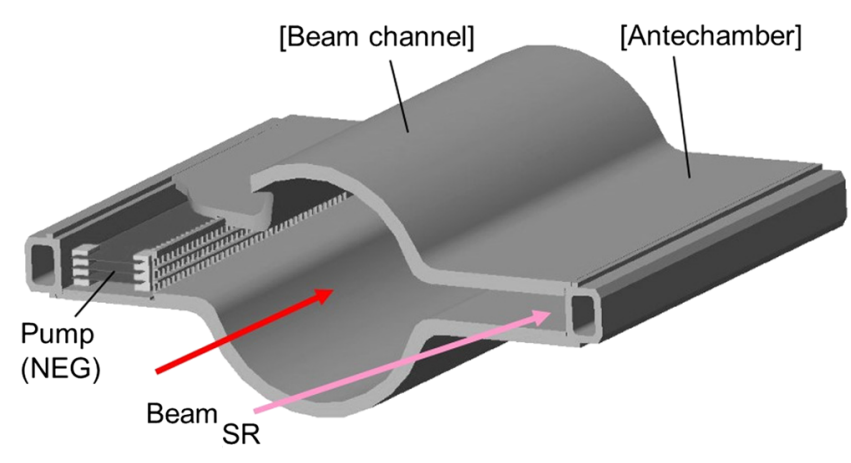

FIG. 2. Typical cross section of a beam pipe at arc sections for LER.

channel $[33,34,42,48,49,54]$ along with a TiN film coating, in order to further reduce the SEY. Instead of the TiN film coating, clearing electrodes $[38,39,47,55]$ were installed in the beam pipes for wiggler magnets, and these also have antechambers. The beam pipes for wiggler magnets were made of copper to manage the intense synchrotron radiation (SR) power. Approximately $90 \%$ of the beam pipes in the ring possessed antechambers and the TiN film coating. $B_{z}$ values of up to several millitesla $[14,19,50,56-58]$ were applied by solenoids to the beam pipes in the drift spaces between electromagnets, such as quadrupole or bending magnets, in the ring.

In Table I, the circles indicate the countermeasures applied in each major section. The electron density $\left(n_{e}\left[\mathrm{~m}^{-3}\right]\right)$ expected in the case of circular beam pipes (copper) and those with the above countermeasures are presented in the table. For the antechamber scheme, coating, solenoid (i.e., $B_{z}$ ), grooved surface, and clearing electrode, the effectiveness in terms of reducing $n_{e}$ is assumed to be $1 / 5,3 / 5,1 / 50,1 / 2$, and $1 / 100$, respectively, on the basis of previous experimental results $[22,24]$. Note here that these effectiveness factors are assumed to be independent of each other. If there are multiple countermeasures, these factors are simply multiplied. For example, if we use both, the antechamber and TiN film coating, the total effectiveness factor should be $1 / 5 \times 3 / 5=3 / 25$.

With all these countermeasures, an $n_{e}$ value of approximately $2 \times 10^{10} \mathrm{~m}^{-3}$ was expected at the design beam parameters, i.e., a beam current of $3.6 \mathrm{~A}$ at a bunch fill pattern of one train of 2500 bunches with a bunch spacing of two rf buckets (hereafter referred to as $1 / 2500 / 2 \mathrm{RF}$ ). This value of $n_{e}$ is sufficiently lower than the $n_{e \text { th }}$ value, $3 \times 10^{11} \mathrm{~m}^{-3}$. Note that $B_{z}$ at drift spaces was not prepared before phase-1 commissioning, because the maximum stored beam current was not expected to be so high during this phase, i.e., approximately $1 \mathrm{~A}$ at the maximum, and the ECE was not anticipated to occur.

The $n_{e}$ around the beam orbit in an Al-alloy beam pipe with antechambers was measured via electron current monitors, which were also used in the previous KEKB experiments [59]. Two electron monitors were set up at the bottom of the beam channel of a test beam pipe. The voltage applied to the electron collector was $100 \mathrm{~V}$, while that applied to the grid (repeller) varied from 0 to $-500 \mathrm{~V}$. These two electron monitors were attached to the same beam pipe: one in the region with the TiN film coating (as in the other typical beam pipes in the ring) and one in the region without the TiN film coating (i.e., bare Al-alloy surface). The test beam pipe was placed in an arc section of the ring. The line density of photons of the SR was $1 \times 10^{15}$ photons $\mathrm{s}^{-1} \mathrm{~m}^{-1} \mathrm{~mA}^{-1}$, i.e., 0.16 photons positron ${ }^{-1} \mathrm{~m}^{-1}$. This line density was almost the same as the average value in the arc sections.

\section{ECE IN PHASE-1 COMMISSIONING}

\section{A. ECE in the early stages}

In spite of the countermeasures described above, the ECE was first observed in phase- 1 commissioning under a beam
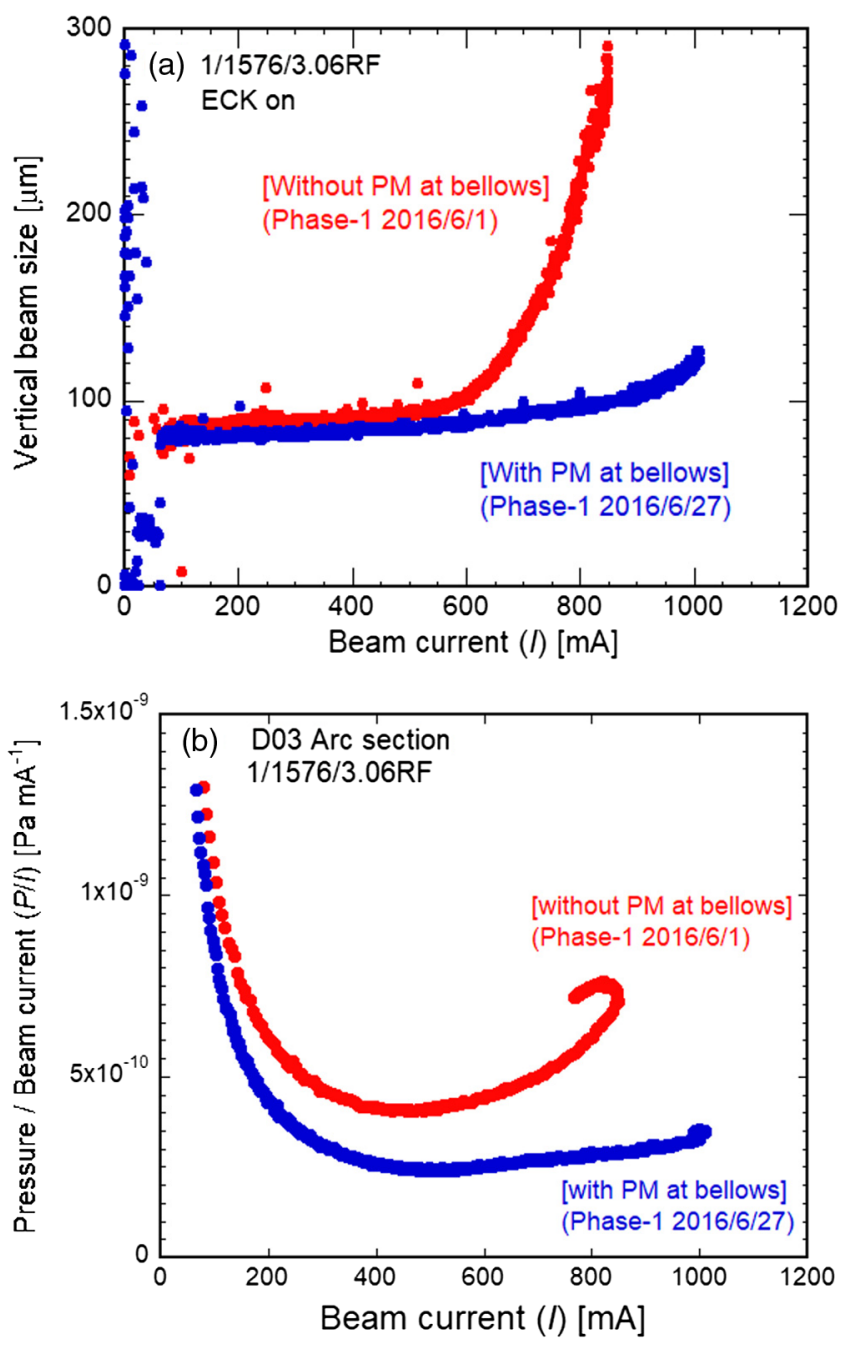

FIG. 3. Measured behaviors of (a) vertical beam size and (b) pressure in an arc section divided by beam current $(P / I)$ against beam current $(I)$ with and without permanent magnet (PM) units on Al-alloy bellow chambers for a bunch fill pattern of 1/1576/3.06RF. 
current $(I[\mathrm{~mA}])$ of approximately $600 \mathrm{~mA}$ at a bunch fill pattern of $1 / 1576 / 3.06 \mathrm{RF}[60,61]$. The vertical beam size began to blow up from this value of $I$, as shown in Fig. 3(a) (without PM at bellows). In this figure, ECK (emittance control knob) is a tool used to adjust the beam emittance via two skew-type quadrupole magnets in the ring. The ECK was active in this case, but had little effect on the behavior of the vertical beam size at $I$ values above $600 \mathrm{~mA}$. The pressure behavior in an arc section $(P[\mathrm{~Pa}])$ divided by $I, P / I$ $\left[\mathrm{Pa} \mathrm{mA}{ }^{-1}\right]$, with respect to $I$ is presented in Fig. 3(b) (without $\mathrm{PM}$ at bellows). Note that high $P / I$ at low $I$ is caused by the base pressures. If the gas load comes solely from photodesorption by SR, $P / I$ should be a constant because the number of photons is proportional to $I$. As shown in Fig. 3(b), however, $P / I$ increased with $I$ because of electron-stimulated gas desorption caused by the multipactoring of electrons [62-64]. This abnormal blowup of beam size and the increase in pressure are typical ECE phenomena.

The blowup of the vertical beam size for bunch fill patterns of 4/150/2RF, 4/150/3RF, 4/150/4RF, and 4/150/6RF is shown in Fig. 4(a) as a function of the current linear density $\left(I_{d}\left[\mathrm{~mA} \mathrm{bunch}^{-1} \mathrm{rf} \mathrm{bucket}^{-1}\right]\right)$, that is, the bunch current ( $I_{\text {bunch }}\left[\mathrm{mA} \mathrm{bunch}^{-1}\right]$ ) divided by the bunch spacing. The threshold of $I_{d}\left(I_{d_{\mathrm{th}}}\left[\mathrm{mA} \mathrm{bunch}{ }^{-1} \mathrm{rf} \mathrm{bucket}^{-1}\right]\right)$ at which the ECE occurs, i.e., the blowup of beam size begins, was approximately $0.1-0.12 \mathrm{~mA}$ bunch $^{-1} \mathrm{rf} \mathrm{bucket}^{-1}$. As the $B_{z}$ was not applied to the beam pipes during phase-1 commissioning, as described before, the excitation of the ECE was an undeniable possibility. However, the threshold beam current for exciting ECE was much lower than expected.

It was finally found that this ECE was caused by the electrons in the Al-alloy bellows chambers without TiN film coating. These are $200 \mathrm{~mm}$ long and are located, on average, every $3 \mathrm{~m}$ around the ring. There are approximately 830 bellows chambers in total, occupying $~ 5 \%$ of the circumference of the ring. However, the value of $n_{e}$ in the test beam pipe at the region without the TiN film coating was found to be on the order of $10^{12} \mathrm{~m}^{-3}$, which is more than 10 times greater than the $n_{e_{\mathrm{th}}}$ value of $3 \times 10^{11} \mathrm{~m}^{-3}$.

To counteract the ECE, two units (type-1 units) of permanent magnets (PMs) were placed at the top and bottom of each Al-alloy bellows chamber, as shown in Fig. 5. One type-1 PM unit had eight PMs attached to a Cshaped iron plate (yoke) [60]. One PM had a diameter of $30 \mathrm{~mm}$ and a magnetic field strength at the surface of approximately $85 \mathrm{mT}$. A $B_{z}$ value of approximately $10 \mathrm{mT}$ was formed in most regions of the PM units, although the polarity reversed locally close to the PMs [60] (Fig. 7 in Sec. IV). After attaching the PM units to all Al-alloy bellows chambers, the abnormal blowup of beam size and increase in $P / I$ disappeared at $I$ values of $600-700 \mathrm{~mA}$, as shown in Fig. 3(a) (with PM at bellows) and Fig. 3(b) (with PM at bellows). A simulation by CLOUDLAND [55] indicated that the $n_{e}$ value around the beam orbit in the Al-alloy
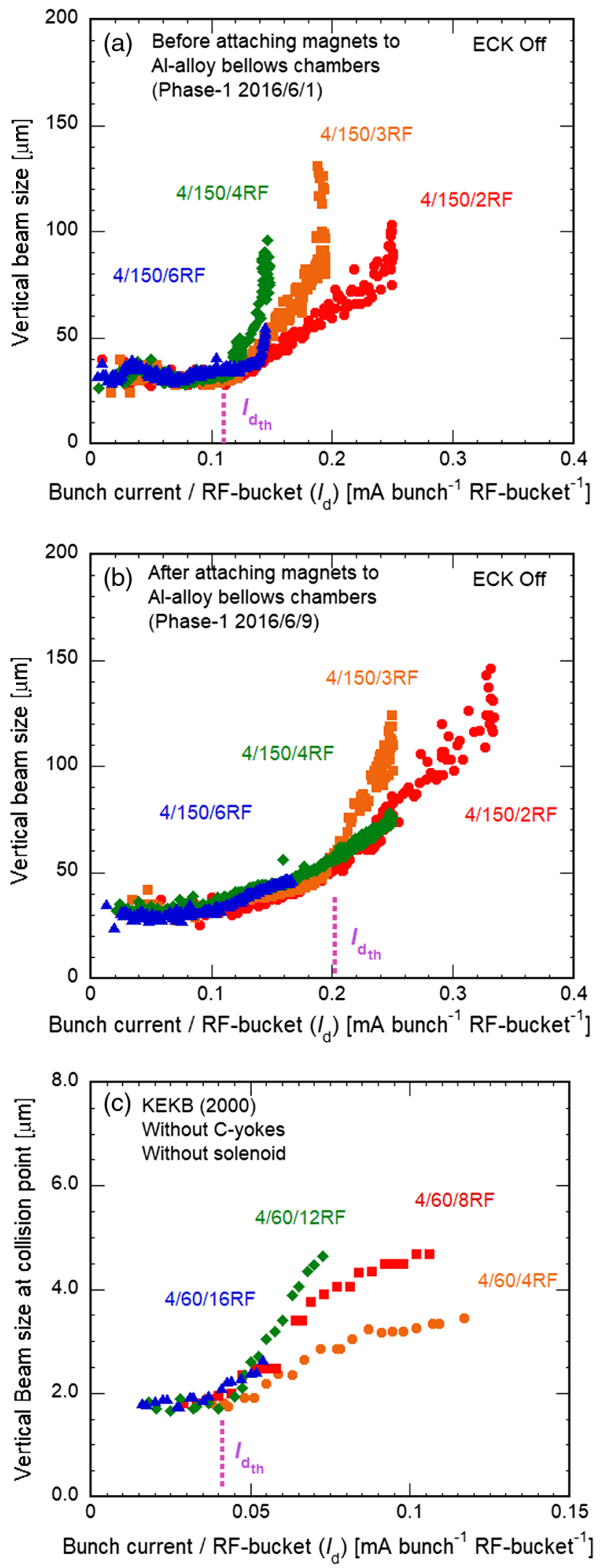

FIG. 4. Measured vertical beam sizes as a function of the current line density $\left(I_{d}\right)$ for several bunch fill patterns measured (a) before and (b) after attaching PM units to Al-alloy bellow chambers in phase- 1 commissioning, and (c) in the early stage of the KEKB era. 
bellows chamber with the PM units should be of the order of $10^{10} \mathrm{~m}^{-3}$, even for the design beam parameters, that is, a beam current of $3.6 \mathrm{~A}$ at a bunch fill pattern of $1 / 2500 / 2 \mathrm{RF}$; here, the maximum SEY $\left(\delta_{\max }\right)$ was assumed to be 2.0, which is a typical value for bare Al-alloy surfaces (see Sec. VIC).

\section{B. ECE at high current}

As described in the previous section, the ECE caused by the electron cloud in the Al-bellows chambers was suppressed by attaching PM units. However, the ECE began to appear again at $I$ values of approximately $900 \mathrm{~mA}$ under a bunch fill pattern of $1 / 1576 / 3.06 \mathrm{RF}$. The blowup of the beam size started from $I=900 \mathrm{~mA}$, as shown in Fig. 3(a) (with PM at bellows). Figure 4(b) shows the dependence of the vertical beam size on $I_{d}$ for bunch fill patterns of 4/150/2RF, 4/150/3RF, 4/150/4RF, and 4/150/6RF. The $I_{d_{\mathrm{th}}}$ value was $0.2 \mathrm{~mA}$ bunch $^{-1} \mathrm{rf} \mathrm{bucket}^{-1}$ for two and three rf-bucket spacings, corresponding to $I$ values of approximately $900 \mathrm{~mA}$ for a bunch fill pattern of $1 / 1576 / 3.06 R F$. Furthermore, the modes of the transverse coupled bunch instabilities (TCBI) were measured and analyzed using a bunch-by-bunch beam feedback system [65]. Unstable modes excited by the electrons in the drift spaces were clearly detected, with frequencies lower by 400-700 times the revolution harmonics with respect to the rf frequency [65-68].

The value of $n_{e}$ in the test beam pipe at the region with the TiN film coating was observed to be close to the $n_{e_{\text {th }}}$ value, that is, approximately $3 \times 10^{11} \mathrm{~m}^{-3}$. The value of $n_{e}$ expected from the tune shift, i.e., $4 \times 10^{11} \mathrm{~m}^{-3}$, was in good agreement with that directly measured in the test chamber at the region with the TiN film coating [68]. Furthermore, the PM units with iron yokes, similar to those used for Al-alloy bellows chambers (Fig. 5), were partially attached for a test around several beam pipes at drift spaces. As a result, the abnormal increase in pressure was suppressed.

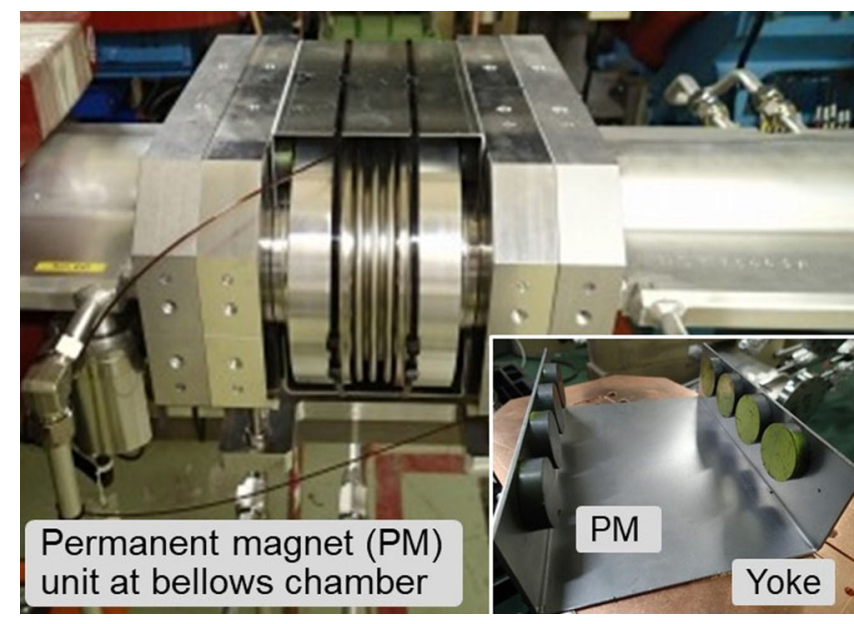

FIG. 5. PM units attached to an Al-alloy bellows chamber.
From these observations, the electron cloud was considered to exist in the beam pipes at drift spaces. Note that no abnormal increase in pressures was observed in the straight sections, including the wiggler magnet sections.

At this point, approximately $90 \%$ of the beam pipes in the LER had antechambers and the TiN coating. Note that the $I_{d_{t h}}$ value of $0.2 \mathrm{~mA}$ bunch $^{-1} \mathrm{rf} \mathrm{bucket}^{-1}$, after suppressing ECE through the Al-alloy bellows chambers, is much higher than that in the early stages of KEKB without any countermeasures, that is, $0.04 \mathrm{~mA}$ bunch $^{-1} \mathrm{rf} \mathrm{bucket}^{-1}$ [Fig. 4(c)] [20,50]. The beam pipes and bellows chambers of KEKB had a simple circular cross section and were made of pure copper or stainless steel without any inner coating. Furthermore, $B_{z}$ was not applied at that time. The $I_{d_{t h}}$ in SuperKEKB was approximately 5 times that in KEKB. This indicated that the antechambers and TiN coating in SuperKEKB effectively suppressed the ECE to some extent. At the same time, however, the excitation of the ECE showed that the countermeasures in phase-1 commissioning were still insufficient; therefore, additional countermeasures against the ECE were prepared before the next commissioning phase. Furthermore, it was necessary to reevaluate the effectiveness of the antechambers and TiN film coating in the real beam pipe to check whether they were working as expected.

\section{ADDITIONAL COUNTERMEASURES}

As additional countermeasures against the ECE, PM units and solenoids were attached to most of the beam pipes at drift spaces in LER. The PM units with $\mathrm{C}$-shaped iron yokes (type-1 units, similar to those applied to Al-alloy bellows chambers), which produce a $B_{z}$ value of approximately $6 \mathrm{mT}$, were placed in sequence one after the other around the beam pipe, as shown in Fig. 6. The axial distributions of the vertical and axial components of the magnetic field ( $B_{y}$ and $\left.B_{z}\right)$ at the top surface, and $B_{z}$ at the center of the beam channel in the type-1 unit are shown in Fig. 7(a). A simulation by the CLOUDLAND code under a magnetic field configuration similar to that in Fig. 7(a)

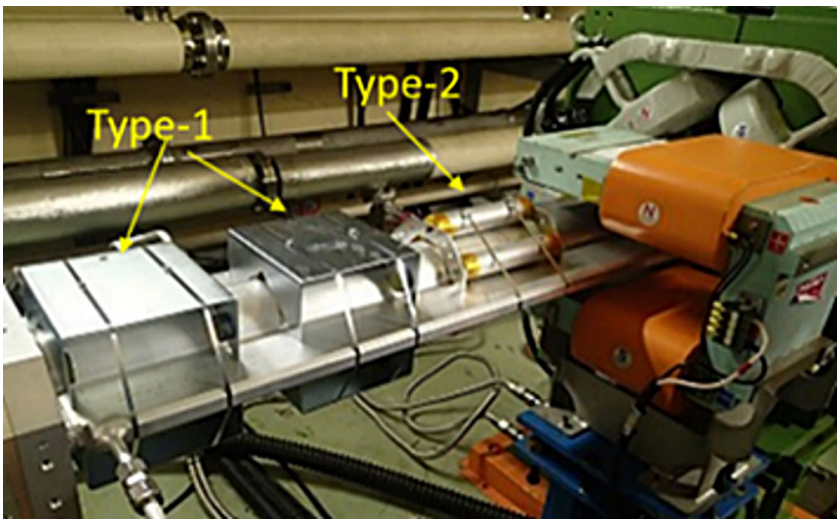

FIG. 6. Type-1 and type-2 units at drift spaces. 

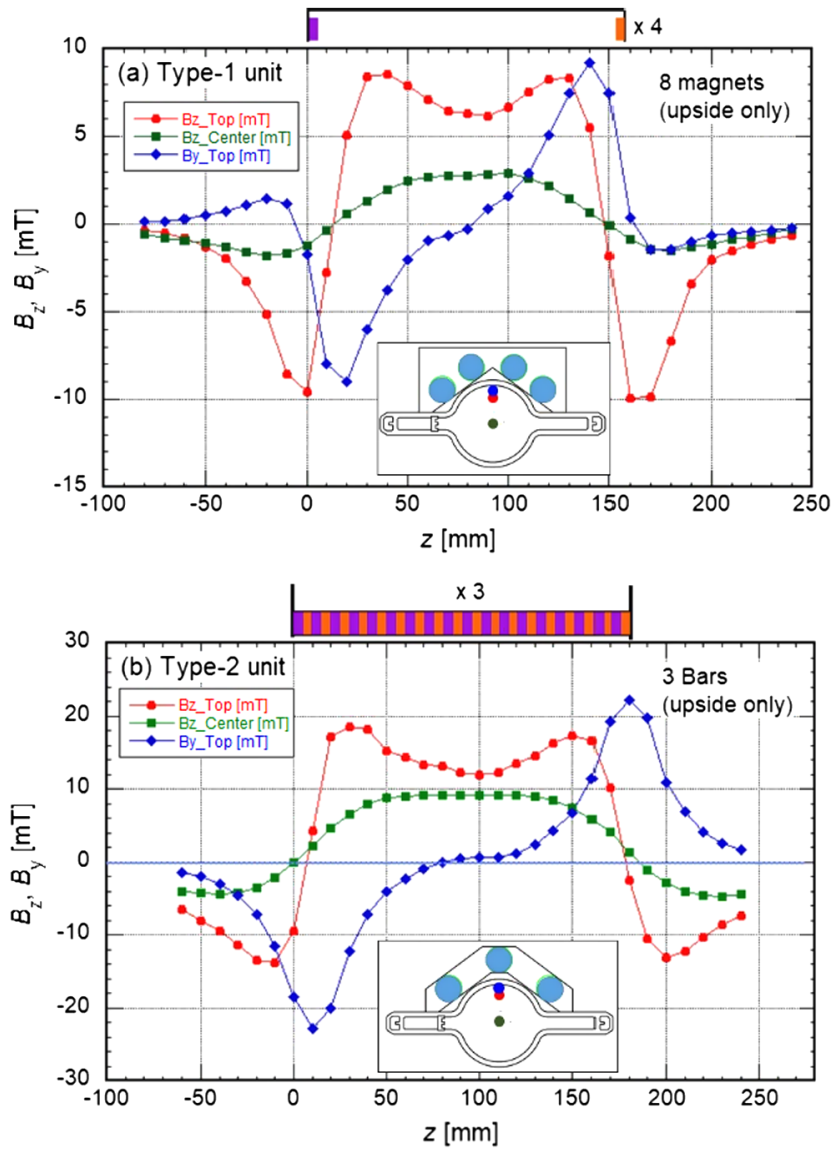

FIG. 7. Axial distributions of the measured vertical and axial components of the magnetic field $\left(B_{y}\right.$ and $\left.B_{z}\right)$ at the top surface, and $B_{z}$ at the center of the beam channel in (a) type-1 and (b) type-2 unit.

indicated that $n_{e}$ around the beam orbit in the units would be reduced to approximately $1 / 10$ of $n_{e_{\text {th }}}$ under the design beam parameters, as presented in Fig. 8. However, the type-1 unit cannot be used near electromagnets such as quadrupoles and sextupoles, because the iron yokes affect their magnetic fields. Therefore, another type of PM unit (type-2 unit), consisting of Al-alloy cylinders with PMs

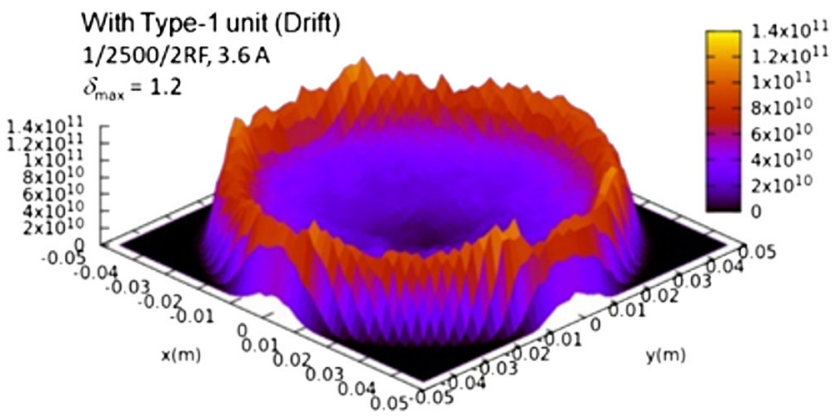

FIG. 8. Simulation result of the electron density in a beam pipe with a type- 1 unit for a beam current of $3.6 \mathrm{~A}$ at a bunch fill pattern of $1 / 2500 / 2 \mathrm{RF}$.

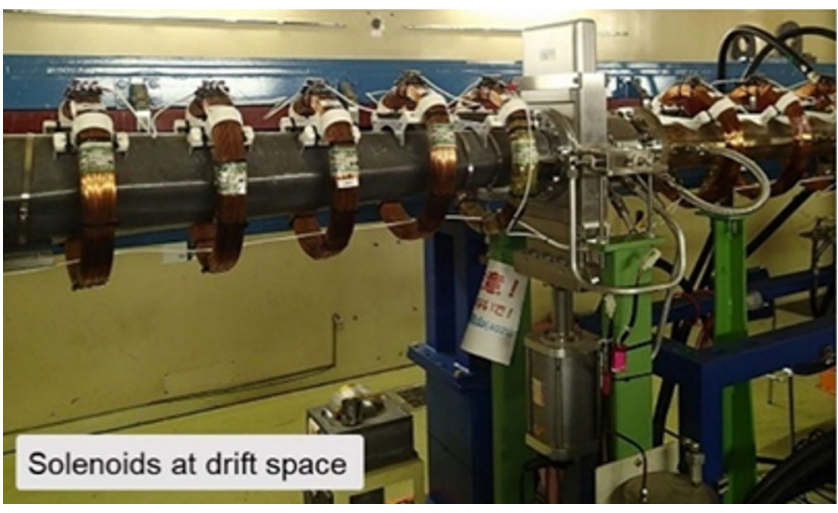

FIG. 9. Solenoids at drift spaces.

inside and Al-alloy supports, was placed near to the electromagnets, as shown in Fig. 6. The axial distributions of $B_{y}$ and $B_{z}$ at the top surface, and $B_{z}$ at the center of the beam channel in the type-2 unit are shown in Fig. 7(b). The value of $B_{z}$ inside the type- 2 units is approximately $10 \mathrm{mT}$. For the beam pipes that had been used since the KEKB era, where the solenoids were already wound, the solenoids were revived, as shown in Fig. 9 [50,57,58].

The magnetic fields generated by these type- 1 and type- 2 units for the beam pipes are not uniform in the beam direction, as indicated in Figs. 7(a) and 7(b). Consequently, some electrons will be trapped in the magnetic field near the pipe wall, which should be included in the high electron density region in Fig. 8. The effect of these trapped electrons on the beam dynamics has not been observed so far [69].

The dependence of $n_{e}$ on $B_{z}$ in the type-1-like magnetic field was calculated for several $\delta_{\max }$ at $I=4$ A with two rf bucket spacings, and the results are presented in Fig. 10.

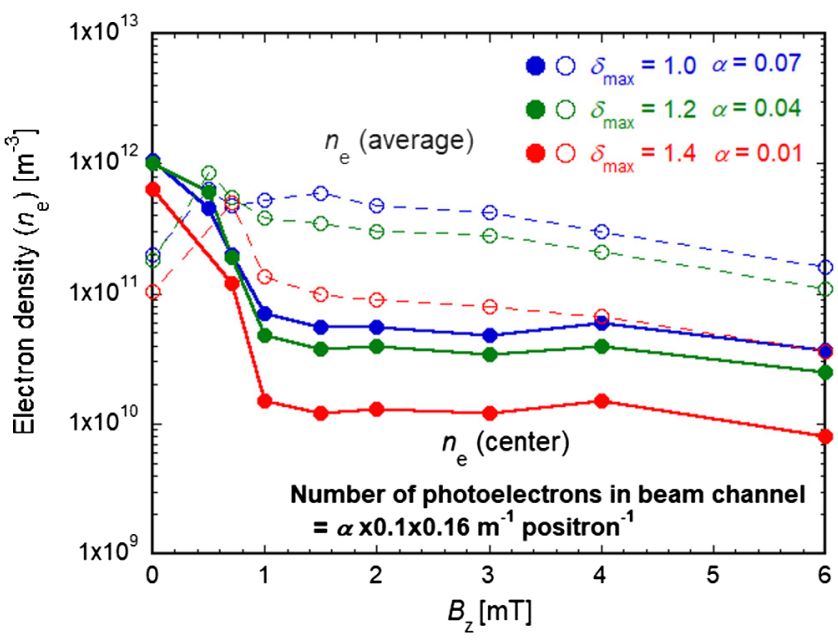

FIG. 10. Measured electron density $\left(n_{e}\right)$ at the center and the average of a beam channel in type- 1 units as a function of the magnetic field in the beam direction $\left(B_{z}\right)$ for several maximum $\operatorname{SEY}\left(\delta_{\max }\right)$ and $\alpha$ (see Sec. V). 
The number of photoelectrons was changed according to the value of $\delta_{\max }$ (see Sec. VI for details). The value of $n_{e}$ was found to be less than $1 \times 10^{11} \mathrm{~m}^{-3}$ when $B_{z}$ was greater than $1 \mathrm{mT}$. Before commencing phase- 2 commissioning, approximately 86\% of the drift spaces (approximately $2 \mathrm{~km}$ in total) was covered with $B_{z}$ of strengths greater than $2 \mathrm{mT}$.

\section{ECE IN PHASE-2 COMMISSIONING}

During phase-2 commissioning from March-July 2018, the vertical beam sizes, pressures, and unstable modes of TCBI were measured as during phase- 1 commissioning.

Figure 11 shows the dependence of the vertical beam size on $I_{d}$ for bunch fill patterns of 4/120/2RF, 4/120/3RF, and 4/120/4RF. As shown in Fig. 11, blowup is not observed until $I_{d}$ reaches $0.4 \mathrm{mAbunch}^{-1} \mathrm{rf} \mathrm{bucket}^{-1}$. The value of $I_{d_{\mathrm{th}}}$ required to excite the ECE increased by a factor of at least 2 relative to the phase- 1 commissioning [Fig. 4(b)].

Figure 12 shows the behavior of $P / I$ at arc sections against $I$ for bunch fill patterns of two rf bucket spacings during phase- 1 and phase- 2 commissioning. As shown in Fig. 12, during phase-1 commissioning, $P / I$ increased with $I$ at values greater than $300 \mathrm{~mA}$. However, $P / I$ remained almost constant as $I$ increased during phase- 2 commissioning. This signifies relatively little multipactoring of electrons. Note that $P$ itself decreased in phase-2 commissioning compared with phase- 1 commissioning because of the vacuum scrubbing [70].

The modes and growth rates of the TCBI were again measured and analyzed [68]. The modes excited by the electrons at the drift space were not observed, even at an $I_{d}$

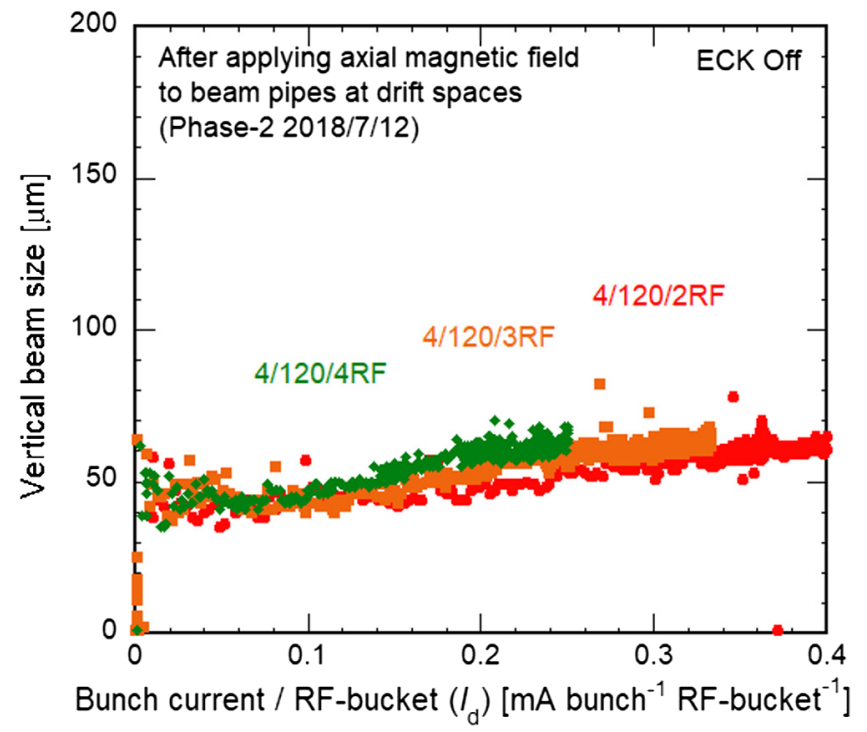

FIG. 11. Measured vertical beam sizes as a function of the current linear density $\left(I_{d}\right)$ for several bunch fill patterns during phase-2 commissioning.

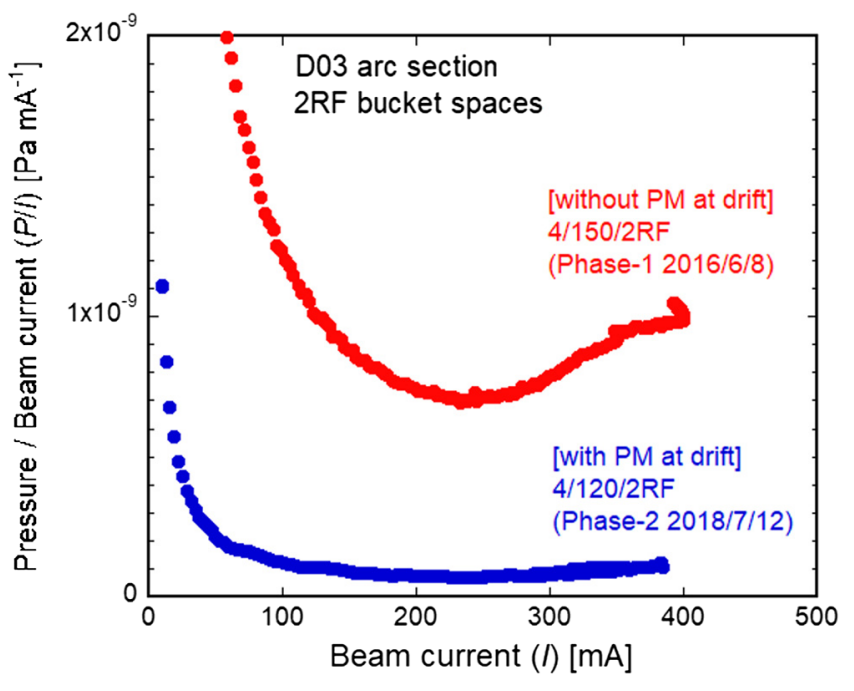

FIG. 12. Measured behavior of pressure in an arc section divided by the beam current $(P / I)$ against the beam current (I) during phase-1 and phase-2 commissioning.

value of $0.2 \mathrm{~mA}$ bunch $^{-1} \mathrm{rf}$ bucket $^{-1}$. Instead, the modes excited by electrons near the inner wall that were trapped by $B_{z}$ were detected, that is, the modes slightly above those corresponding to the bunch filling harmonics. Furthermore, the growth rates of the unstable modes were much lower than those observed in phase- 1 commissioning.

The value of $n_{e}$ in the test beam pipe at the region with the TiN film coating was unchanged from that in phase-1 commissioning, i.e., the beam pipe conditions, such as $\delta_{\max }$ of the TiN film, were the same as in phase- 1 commissioning, except for $B_{z}$ applied to beam pipes at the drift spaces.

From these observations, the additional countermeasures, that is, the application of $B_{z}$ by PM units and solenoids, were found to be working well in terms of suppressing the ECE during phase-2 commissioning.

\section{REEVALUATION OF THE EFFECTS OF ANTECHAMBERS AND TIN COATING}

First, as a measure of the effectiveness of a beam pipe with an antechamber with regards to the suppression of photoelectrons, the rate of reduction in the number of photoelectrons in the beam channel, relative to a simple circular beam pipe, is defined as

$$
\alpha \equiv \frac{p_{b}+\beta \times p_{a}}{p_{b}+p_{a}},
$$

where $p_{b}$ and $p_{a}$ are the numbers of photoelectrons generated in the beam channel and antechamber, respectively. The total number of photoelectrons at the location is, therefore, $p_{b}+p_{a} . \beta$ is the probability that the electrons in the antechamber pass into the beam channel. A small value of $\alpha$ would indicate that the antechambers are highly effective. 
The maximum SEY, $\delta_{\max }$, was used here to measure the effectiveness of the TiN film coating with regard to the suppression of secondary electrons. Note, however, that while $\delta_{\max }$ is the most important parameter, it is not the only one that determines $n_{e}$. For example, the electron reflectivity at very small energies is also a sensitive parameter [71].

\section{A. Calculation of $\beta$}

First, $\beta$ in Eq. (1) was estimated from a simulation code PEI [4] to calculate the motion of electrons. The electric field due to an electron cloud in the beam channel was calculated by a band-matrix solver. The force from a positron beam was calculated using the Bassetti-Erskine equation or the beam potential equation for electrons inside or outside a diameter of $10 \sigma_{x}$, respectively, where $\sigma_{x}$ is the transverse beam size. Photoelectrons were assumed to be generated only at the innermost wall of the antechamber. Furthermore, because the wall is a technical surface and is also roughened by glass bead blasting (GBB) $[24,44]$, the emission angle of photoelectrons was assumed to obey the cosine law [72-74].

In cases where the space charge effect and the reflection of electrons were neglected, i.e., low $n_{e}$, the value of $\beta$ was approximately 0.07 . In contrast, when the space charge was taken into account assuming $I_{\text {bunch }}=1 \mathrm{~mA} \mathrm{bunch}^{-1}$, a bunch spacing of three rf buckets, $\delta_{\max }=1.2$, and a reflection rate of electrons of 0.7 , i.e., high $n_{e}$, the value of $\beta$ was $0.03-0.04$. In the following discussions, a $\beta$ value of 0.05 is assumed as a typical value.

\section{B. Relationship between $\alpha$ and $\delta_{\max }$ under a constant $\boldsymbol{n}_{\boldsymbol{e}}$}

Figure 13 shows $n_{e}$ as a function of $\delta_{\max }$ for $\alpha$ values of $0.01,0.03,0.06$, and 0.09 at a bunch fill pattern of $1 / 150 / 2 \mathrm{RF}$, calculated by the simulation code PyECLOUD [75], using a circular beam pipe. The line density of photons was $1 \times 10^{15}$ photons $\mathrm{s}^{-1} \mathrm{~m}^{-1} \mathrm{~mA}^{-1}$, i.e., 0.16 photons positron ${ }^{-1} \mathrm{~m}^{-1}$. The value of $I_{\text {bunch }}$ was $0.8 \mathrm{~mA} \mathrm{bunch}^{-1}$. For $\delta_{\max }$ values of less than $1.2, n_{e}$ was of the order of $10^{10}-10^{11} \mathrm{~m}^{-3}$, where the space charge effect is small, and is almost proportional to $\alpha$ for a constant $\delta_{\max }$. In contrast, for $\delta_{\max }$ values greater than $1.5, n_{e}$ was of the order of $10^{12}-10^{13} \mathrm{~m}^{-3}$, where the space charge limits $n_{e}$, which is independent of both $\alpha$ and $\delta_{\max }$. As described above, the value of $n_{e_{\text {th }}}$ for our case is approximately $3 \times 10^{11} \mathrm{~m}^{-3}$. Therefore, $n_{e}$ values of the order of $n_{e_{\text {th }}}$ values were not only determined by the SEY (i.e., $\delta_{\max }$ ), but also by the number of photoelectrons in the beam channel (i.e., $\alpha$ ), and $n_{e}$ was almost proportional to $\alpha$ for a fixed $\delta_{\max }$.

From the observations made during phase- 1 commissioning, the ECE was observed, i.e., the beam size blowup started, at an $I$ value of approximately $900 \mathrm{~mA}$ for a bunch fill pattern of $1 / 1576 / 3.06 \mathrm{RF}$. This means that $n_{e}$ should be approximately $3 \times 10^{11} \mathrm{~m}^{-3}$ for these beam parameters.

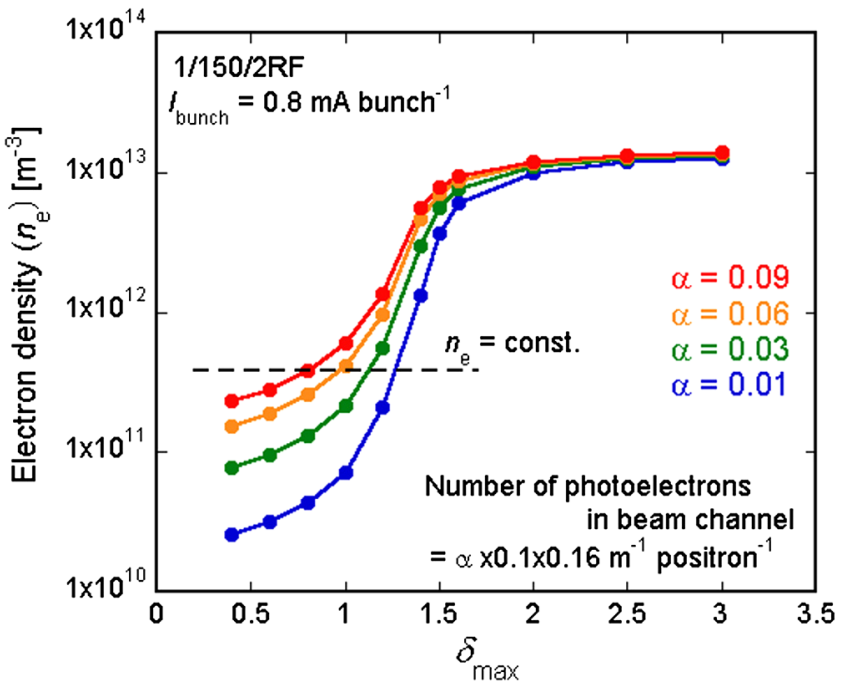

FIG. 13. Dependence of calculated electron density $\left(n_{e}\right)$ on the maximum SEY $\left(\delta_{\max }\right)$ for several $\alpha$, that is, the number of photoelectrons in a beam channel, at a bunch fill pattern of $1 / 150 / 2 \mathrm{RF}$. The dotted line denotes the combination of $\alpha$ and $\delta_{\max }$ that gives a constant $n_{e}$.

Actually, the measured $n_{e}$ at the region with the TiN film coating in the test beam pipe was almost the same under the same beam parameters $[60,61]$. Under this condition, $\delta_{\max }$ was calculated as a function of the number of photoelectrons in the beam channel using the simulation code CLOUDLAND, where a circular beam pipe was again used as a model. The results are presented in Fig. 14. The value of $\alpha$ corresponding to the number of photoelectrons in the beam channel is also plotted in Fig. 14. Here, the bunch fill pattern was $1 / 150 / 3 \mathrm{RF}$, the number of positrons in a

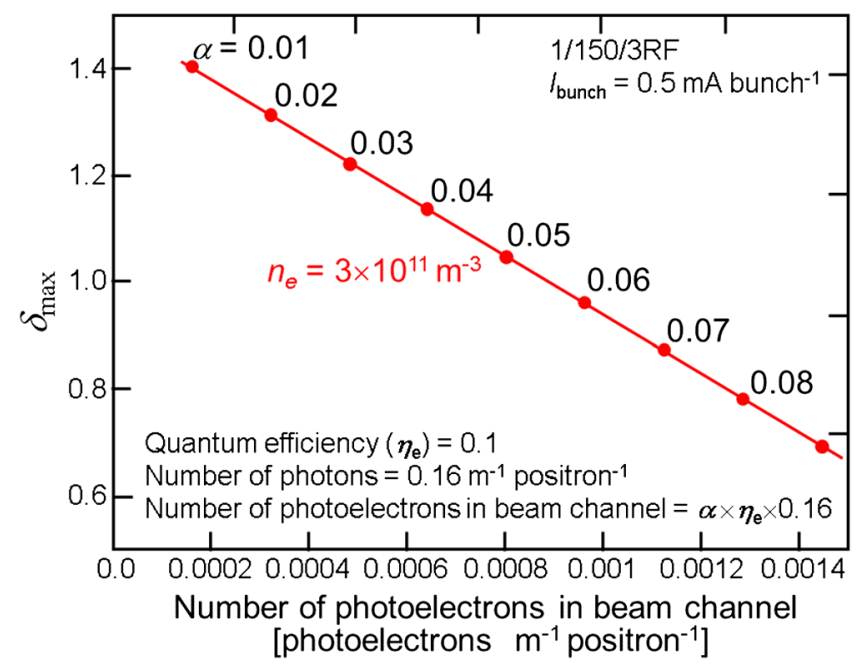

FIG. 14. Calculated results for combinations of $\delta_{\max }$ and number of photoelectrons in a beam channel that give the same electron density $\left(n_{e}\right)$ of $3 \times 10^{11} \mathrm{~m}^{-3}$ at $900 \mathrm{~mA}$ for a bunch fill pattern of $1 / 1576 / 3.06 \mathrm{RF}$. The values of $\alpha$ corresponding to the number of photoelectrons are also given. 
bunch was $3.1 \times 10^{10}$ bunch $^{-1}$ (corresponding to $0.5 \mathrm{~mA} \mathrm{bunch}^{-1}$ ), and the line density of photons from SR was 0.16 photons positron ${ }^{-1} \mathrm{~m}^{-1}$. This line density is equal to the average value in the arc sections, as mentioned above. The quantum efficiency was assumed to be a constant 0.1 . Furthermore, the photoelectrons were emitted uniformly inside the beam channel.

It is interesting that the combinations of $\alpha$ and $\delta_{\max }$ similar to the line in Fig. 14 can be plotted along the dotted line in Fig. 13, although the beam parameters are different. This tendency is a characteristic of $n_{e}$ at this order of magnitude.

If $\alpha$ was estimated from simulations or measurements, $\delta_{\max }$ at the surface could be deduced using Fig. 14. For example, the $\alpha$ value was estimated to be 0.01 from an experiment during KEKB commissioning using a test beam pipe with an antechamber made of pure copper [44]. In this case, $\delta_{\max }$ was estimated to be approximately 1.4 from Fig. 14. This value of $\delta_{\max }$ is higher than the 1.0-1.2 obtained for the TiN film coating after sufficient electron bombardment in a laboratory [46]. Various hypotheses explaining this discrepancy, such as the imperfect TiN film coating, insufficient electron bombardment (i.e., aging), or high pressures in the beam pipe, have been considered, but they do not provide definitive explanations. For example, $n_{e}$ in the test beam pipe at the region with the TiN film coating did not change during phase- 2 commissioning, as described in Sec. V. The pressures in the arc sections during phase-2 commissioning were a similar order of magnitude as those in the laboratory experiments. Therefore, to evaluate the actual $\delta_{\max }$, it is necessary to estimate $\alpha$ in a real beam pipe.

\section{Reevaluation of $\alpha$ and $\delta_{\text {max }}$}

The values of $\alpha$ and $\delta_{\max }$ in the real beam pipe of the ring were reevaluated using four methods, including simulations and dedicated experiments, during phase- 2 commissioning.

\section{From the photon distribution}

Using the cross section and surface roughness of a real beam pipe with antechambers, the number of photons inside the beam pipe was calculated by the simulation code Synrad3D [76]. The innermost wall of the antechamber in the real beam pipe, where the SR is directly irradiated, was roughened by GBB $[24,44]$. The measured roughness in $\mathrm{rms}(\sigma[\mu \mathrm{m}])$ was $11.59 \mu \mathrm{m}$ and the correlation length of the roughness $(T[\mu \mathrm{m}])$ was $47.59 \mu \mathrm{m}$, giving a ratio $T / \sigma$ of 4.11. The inner wall of the beam channel, which is a surface of an extruded Al-alloy pipe, had a $\sigma$ value of $0.647 \mu \mathrm{m}$ and a $T$ value of $9.52 \mu \mathrm{m}$, giving $T / \sigma=14.12$. The distribution of photons absorbed to the inner wall of the beam pipe was calculated under the real layout of electromagnets at the location where $n_{e}$ was measured. The scattered photons from upstream of the location were
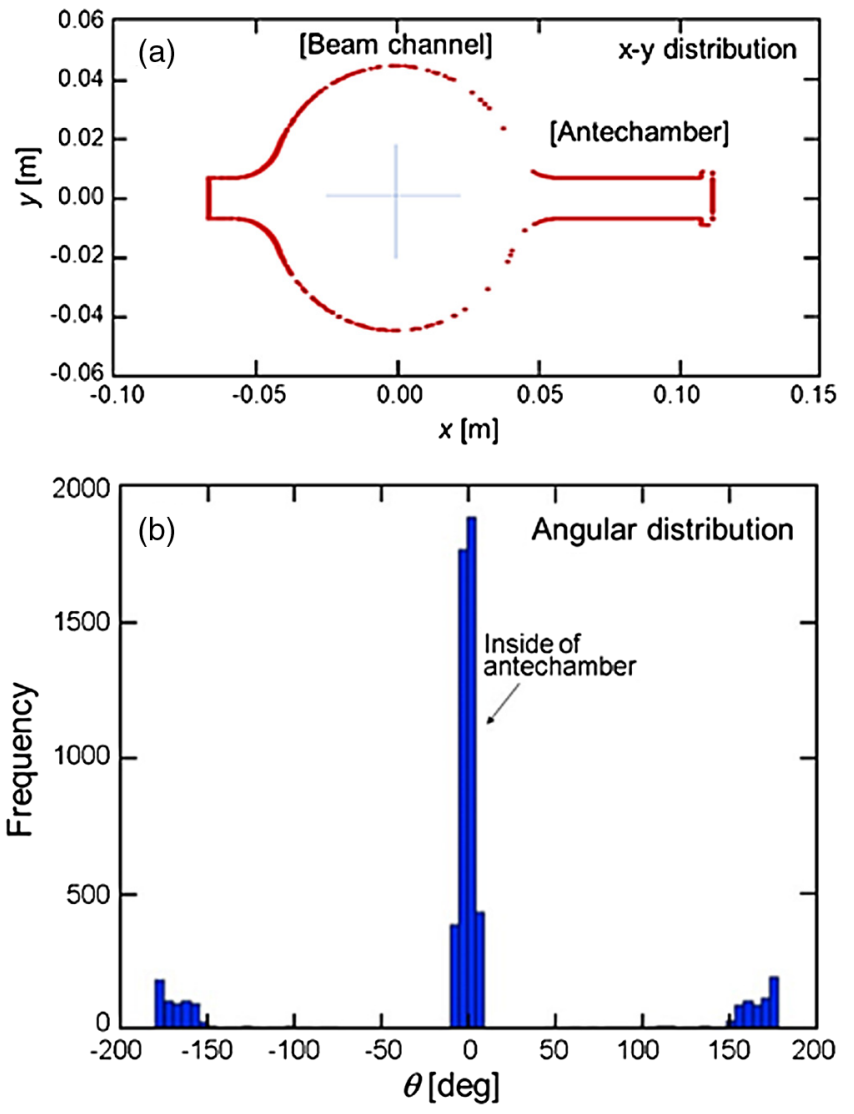

FIG. 15. (a) Positions of absorbed photons along the peripheral of the beam pipe, and (b) their angular distribution, where $0^{\circ}$ corresponds to the inner most side of the antechamber, calculated by the simulation code Synrad3D.

taken into account in the calculation under the assumption that a TiN film with a thickness of $200 \mathrm{~nm}$ was coated on the Al-alloy surface [46].

Figure 15(a) shows the calculated distribution of absorbed photons along the periphery of the cross section, and Fig. 15(b) shows the angular distribution, where $\theta=0$ corresponds to the innermost part of the antechamber. The line density of total photons absorbed at this location was 0.16 photons positron ${ }^{-1} \mathrm{~m}^{-1}$. The line density of photons absorbed in the beam channel was 0.00956 photons positron ${ }^{-1} \mathrm{~m}^{-1}$. The number of photoelectrons can be obtained by multiplying the quantum efficiency with the number of absorbed photons. Assuming a constant value for the quantum efficiency as a function of the photon energy, we have

$$
\frac{p_{b}}{p_{b}+p_{a}}=\frac{0.00956}{0.16}=0.06
$$

Using $\beta=0.05$, a value of $\alpha=0.11$ was calculated from Eqs. (1) and (2). The value of $\delta_{\max }$ was then evaluated as $0.5-0.6$ from the extrapolated line in Fig. 14. This value of 
$\delta_{\max }$ is quite low comparing the data obtained thus far [46]; this will be discussed again in Sec. VID.

\section{From the measured $n_{e}$}

For values of the order of $10^{11} \mathrm{~m}^{-3}, n_{e}$ is almost proportional to the number of photoelectrons in the beam channel for a constant $\delta_{\max }$, as indicated in Fig. 13. The ratio of $n_{e}$ when the number of electrons from the antechambers is negligible, $n_{e 0}$, to that under usual conditions, $n_{e}$, can be computed as follows:

$$
\frac{n_{e 0}}{n_{e}}=\frac{p_{b}}{p_{b}+\beta \times p_{a}} .
$$

Therefore, if $n_{e 0}$ is measured, $\alpha$ can be deduced using Eqs. (1) and (3).

During phase- 2 commissioning, $n_{e 0}$ was measured by attaching weak PMs along the ends of the antechambers of the test beam pipe, as shown in Fig. 16. These magnets generate weak vertical magnetic fields $\left(B_{y}[\mathrm{mT}]\right)$ along the antechamber, trapping the emitted photoelectrons inside. The value of $B_{y}$ near the PMs was approximately $10 \mathrm{mT}$, but that in the beam channel was less than $0.05 \mathrm{mT}$, which is the same order as terrestrial magnetism. In the simulation, $B_{y}$ of this order of magnitude had no effect on $n_{e}$ in the beam channel. Furthermore, $B_{y}$ of this order of magnitude was experimentally found to have little effect on the measurement of $n_{e}$ by the present electron monitors.

The measured $n_{e 0}$ and $n_{e}$ at a bunch fill pattern of $1 / 1576 / 3.06 \mathrm{RF}$ during phase-2 commissioning are presented in Fig. 17. Note that the high $n_{e}$ values at low $I_{\text {bunch }}$ are not reliable, as the volume used in the calculation of $n_{e}$ is so small that the estimation method is no longer valid in principle [59]. The value of $n_{e 0} / n_{e}$ was $1.5 / 3.3$ at an $I_{\text {bunch }}$ value of $0.45 \mathrm{~mA} \mathrm{bunch}^{-1}$. From Eq. (3), the value of $p_{b} / p_{a}$ was calculated to be 0.04 for a $\beta$ value of 0.05 . The value of $\alpha$ was then calculated to be 0.08 using Eq. (1).

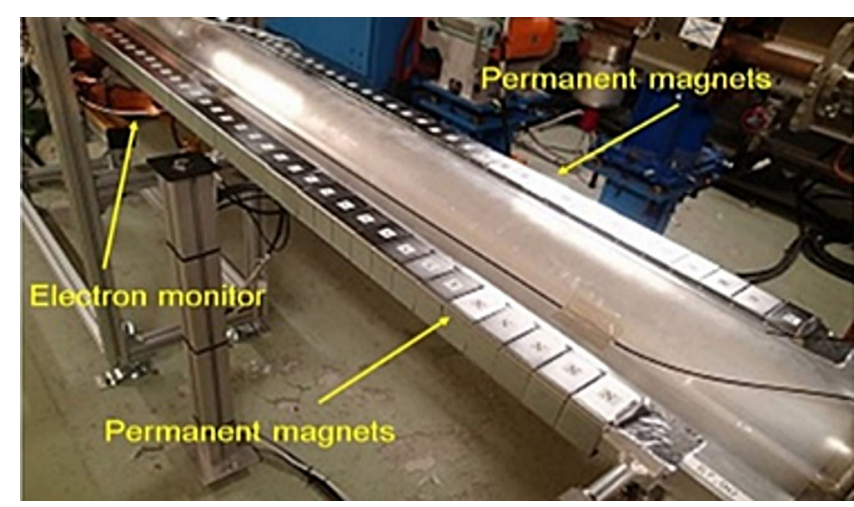

FIG. 16. Weak PMs attached at the antechambers of the test beam pipe with electron monitors to prevent the photoelectrons generated in the antechamber from entering the beam channel.

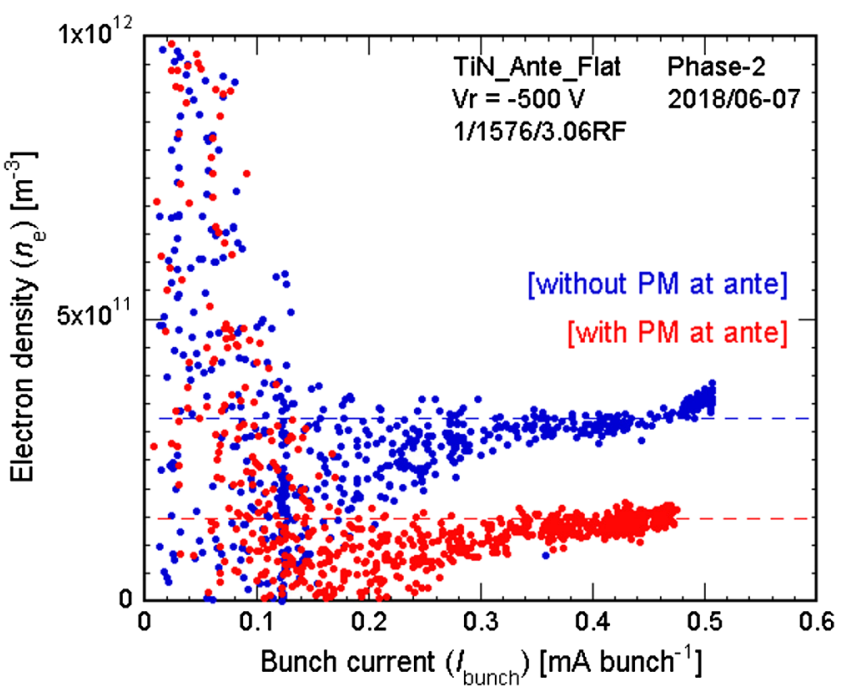

FIG. 17. Measured electron density $\left(n_{e}\right)$ near the beam orbit for the cases with and without PMs in the antechambers.

Consequently, using the relation in Fig. 14, $\delta_{\max }$ was estimated to be approximately $0.7-0.8$.

\section{From the beha vior of $n_{e}$ with respect to $I$}

Both $\alpha$ and $\delta_{\max }$ were estimated from the behavior of the measured $n_{e}$ against $I_{d}$ and compared with the values obtained by simulations. The behavior depends on $\alpha, \delta_{\max }$, and also the bunch fill pattern [75]. Figures 18(a) and 18(b) show the dependencies of $n_{e}$ measured at the region with the TiN film coating in the test beam pipe (without PM units) on $I_{d}$ for bunch fill patterns of $1 / 150 / 2 \mathrm{RF}$ and $1 / 120 / 3 R F$, respectively, during phase-2 commissioning, and also show the dependencies of $n_{e}$ calculated using the simulation code PyECLOUD with a circular pipe model and bunch fill patterns of $1 / 150 / 2 \mathrm{RF}$ and $1 / 150 / 3 \mathrm{RF}$, respectively, for $\left(\delta_{\max }, \alpha\right)=(0.8,0.09),(1.0,0.06),(1.2,0.04)$, and $(1.4,0.01)$. These combinations of $\delta_{\max }$ and $\alpha$ give the same $n_{e}$ value of approximately $3 \times 10^{11} \mathrm{~m}^{-3}$ at an $I_{\text {bunch }}$ value of $0.5 \mathrm{mAbunch}^{-1}$ and a bunch fill pattern of $1 / 150 / 3 R F$, and almost follow the relation indicated by the line in Fig. 14. The calculated behavior of $n_{e}$ was very similar to the measured relations for $\alpha=0.03-0.06$ and $\delta_{\max }=1.0-1.2$. Similar results were obtained for other bunch fill patterns of four rf-bucket spacings.

\section{From the dependence of $n_{e}$ on the train length}

This method is suitable for deducing $\delta_{\max }$. As $n_{e}$ generally increases (builds up) with bunches from the beginning of a bunch train [6,9], its value should depend on the length of the train. The solid lines in Figs. 19(a)-19(f) show the behaviors of $n_{e}$ with respect to $I_{\text {bunch }}$ for bunch fill patterns of $1 / 60 / 2 \mathrm{RF}$ and $1 / 150 / 2 \mathrm{RF}$ and $\delta_{\max }$ values from $0.8-2.0$, respectively, which were calculated by the 

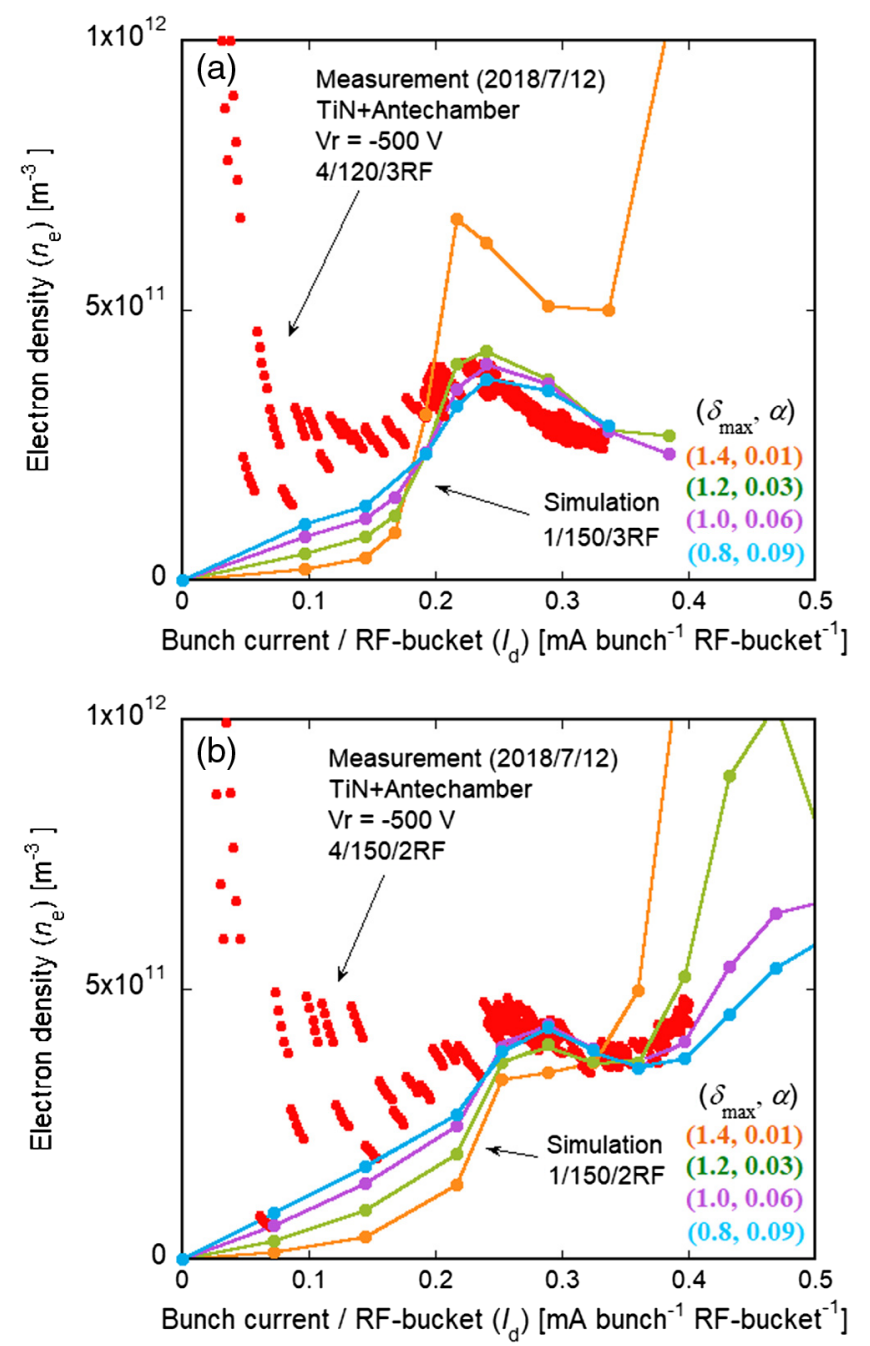

FIG. 18. Measured electron density $\left(n_{e}\right)$ near the beam orbit and calculated density for several combinations of $\left(\delta_{\max }, \alpha\right)$ as a function of the current linear density $\left(I_{d}\right)$ for bunch fill patterns of (a) two and (b) three rf-bucket spacings.

simulation code PyECLOUD. Here $\alpha$ was set to 0.06 as a typical value from methods (i)-(iii) above. Actually, the value of $\alpha$ has little effect on the qualitative behavior of $n_{e}$ against $I_{\text {bunch. }}$. For small $\delta_{\max }\left(\delta_{\max }<1.2\right)$, the multiplication of electrons is small and $n_{e}$ soon becomes saturated at the limit determined by the balance between the generation and loss of electrons. Therefore, the dependence of $n_{e}$ on the train length is weak when the train length is longer than several tens of bunches. For larger $\delta_{\max }\left(1.2<\delta_{\max }<1.6\right)$, $n_{e}$ continues to increase with the number of bunches by multipactoring until the limit determined by the space charge effect. As it takes some time to reach this limit, $n_{e}$ depends on the train length. For larger $\delta_{\max }\left(\delta_{\max }>2.0\right), n_{e}$ soon reaches the limit determined by the space charge effect owing to the strong multipactoring, and then the dependence on the train length again becomes weak. These tendencies are indicated in Fig. 19. Note that the vertical scale in each plot is adjusted to focus on the specific behavior.

The dependence of $n_{e}$ on the train length in the test beam pipe at the region with the TiN film coating was measured during phase- 2 commissioning. The results for bunch fill patterns of $4 / 60 / 2 \mathrm{RF}$ and $1 / 150 / 2 \mathrm{RF}$ are plotted in Fig. 19(b). Comparing the measured results with the calculated ones in the same figure, the behavior of the measured $n_{e}$ gives the best fit when $\delta_{\max }=0.8-1.0$.

For reference, the behavior of the measured $n_{e}$ with respect to $I_{\text {bunch }}$ at the region without the TiN coating (i.e., bare $\mathrm{Al}$ alloy) for these two bunch fill patterns is shown in Fig. 19(f). The behavior of the measured $n_{e}$ fits well for $\delta_{\max } \sim 2.0$.

\section{Results of reevaluation}

The results presented in the previous section can be summarized as follows, and are plotted in Fig. 20: (i) $\alpha=0.11$ and $\delta_{\max }=0.5-0.6$; (ii) $\alpha=0.09$ and $\delta_{\max }=0.7-0.8$; (iii) $\alpha=0.03-0.06$ and $\delta_{\max }=1.0-1.2$; (iv) $\delta_{\max }=0.8-1.0$ (for $\alpha=0.06$ ).

Note that the evaluation by method (i) used the number of photoelectrons calculated from the number of photons assuming a constant quantum efficiency for both the antechamber (rough surfaces and no TiN film coating) and the beam channel (smooth surface and TiN film coating). This is different from methods (ii) and (iii), where the number of photoelectrons is directly included. The difference may explain the relatively high value of $\alpha$ given by method (i). If the quantum efficiency of the TiN film coating is less than that of the $\mathrm{Al}$ surface, for example [45], $\alpha$ should be smaller than the obtained value.

Although the results are relatively scattered, all values of $\alpha$ are greater than that obtained in the KEKB experiments, i.e., 0.01 [44]. This difference can be explained by the following: (a) the location of the experimental setup, i.e., just downstream (KEKB) and $7 \mathrm{~m}$ downstream (SuperKEKB) of a bending magnet, (b) the height of the antechamber, i.e., $18 \mathrm{~mm}$ (KEKB) and $14 \mathrm{~mm}$ (SuperKEKB), (c) the beam pipe material, i.e., copper (KEKB) and Al-alloy (SuperKEKB), (d) the treatment of the innermost surface of the antechamber where the SR is directly irradiated, and so on. Among these, reasons (a) and (b) seem the most plausible. Some photons from upstream should hit the beam channel far downstream of the bending magnets because of the vertical spread and scattering of SR in the real machine. Further investigations are required to clarify the precise reason.

As for $\delta_{\max }$ of the TiN film, the values are close to or somewhat lower than those obtained in the laboratory [46]. The TiN film coating seems to be working as well as expected with regard to reducing the emission of secondary electrons. 

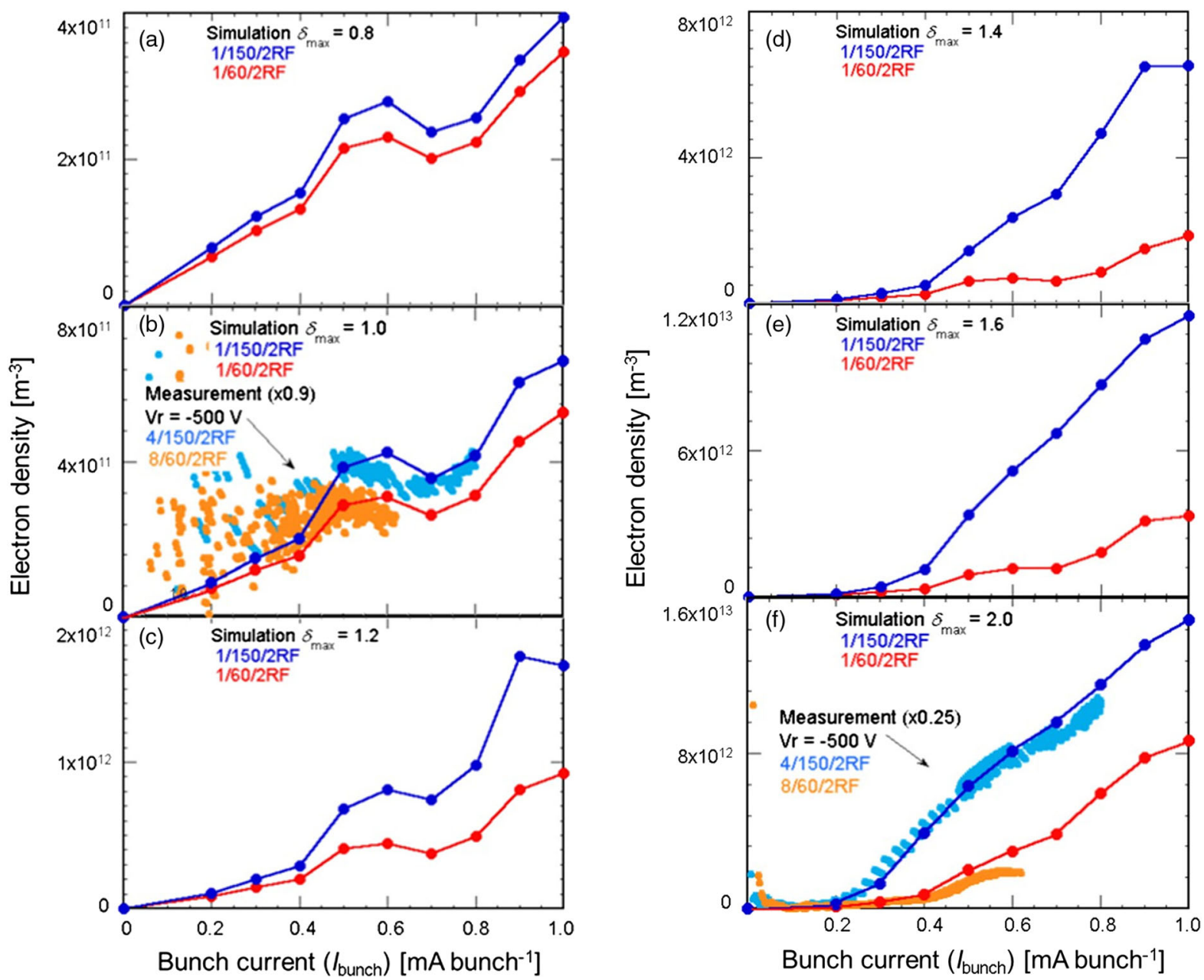

FIG. 19. Calculated behaviors of the electron density $\left(n_{e}\right)$ with respect to the bunch current $\left(I_{\text {bunch }}\right)$ for bunch fill patterns of $1 / 60 / 2 \mathrm{RF}$ and $1 / 150 / 2 \mathrm{RF}$ and $\delta_{\max }$ from (a) 0.8, (b) 1.0, (c) 1.2, (d) 1.4, (e) 1.6, and (f) 2.0, respectively, where $\alpha=0.06$. Vertical scales are adjusted to focus on the specific behavior. Measured behaviors of the electron density $\left(n_{e}\right)$ in the test beam pipe at the region with and without the TiN coating against the bunch current $\left(I_{\text {bunch }}\right)$ at bunch fill patterns of 8/60/2RF and 4/150/2RF are also plotted in (b) and (f), respectively.

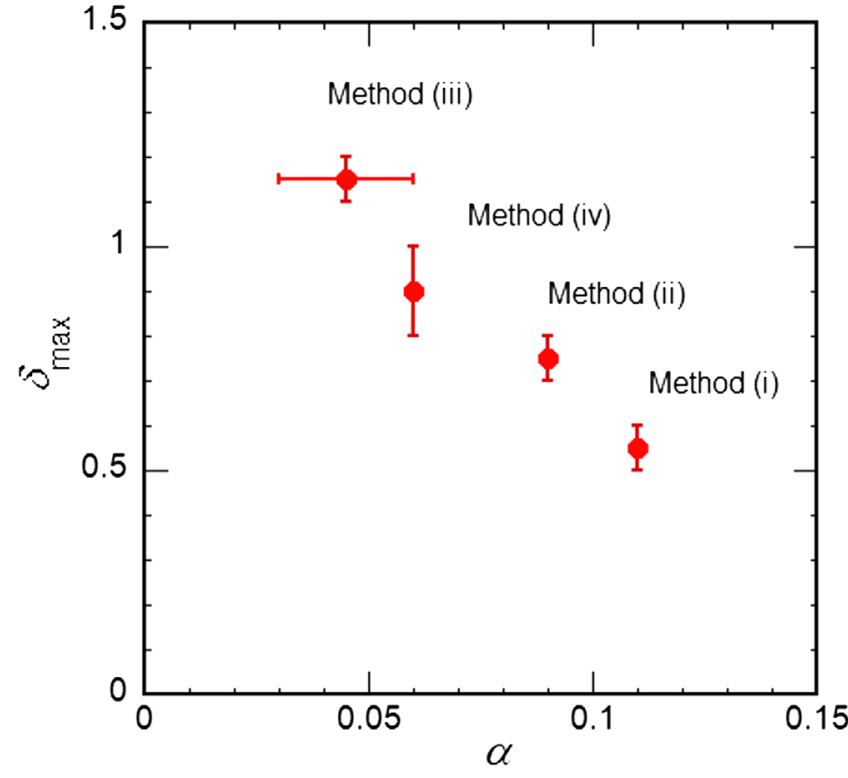

FIG. 20. Summary of the results in Sec. VI.

\section{SUMMARY}

The ECE was excited in the SuperKEKB LER during phase-1 commissioning. The more serious ECE observed in the high-current region was caused by the electron cloud in the beam pipes at drift spaces, which already had the TiN film coating and antechambers. Additional countermeasures, that is, the application of permanent magnet units and solenoids to generate a $B_{z}$ of several tens of $\mathrm{mT}$, worked well during phase- 2 commissioning, and the ECE was not observed until a current linear density of $0.4 \mathrm{mAbunch}^{-1} \mathrm{rf} \mathrm{bucket}^{-1}$. Although the current linear density at the design parameters is $0.7 \mathrm{~mA} \mathrm{bunch}^{-1} \mathrm{rf} \mathrm{bucket}^{-1}$, the countermeasures in SuperKEKB have been working almost as expected.

During phase- 2 commissioning, the effectiveness of the antechamber $(\alpha)$ and TiN film coating $\left(\delta_{\max }\right)$ of the real beam pipe in the ring was reevaluated through simulations and dedicated experiments. The value of $\alpha$ in the real beam pipe was found to be larger than expected. The most plausible reason for this large value is the irradiation of 
photons in the beam channel due to the vertical spread and scattering of SR in the real ring. The value of $\delta_{\max }$, however, decreased to the values obtained in the laboratory. These results indicate the importance of photoelectrons for the ECE in the real machine $[52,76,77]$.

Phase-3 commissioning of SuperKEKB will start in early 2019, and the beam current will be increased further. Before commencing phase- 3 commissioning, PM units will be added with the aim of covering over $90 \%$ of the drift spaces with a $B_{z}$ greater than $2 \mathrm{mT}$. Careful observations of the ECE will continue during phase- 3 commissioning and beyond.

In terms of beam optics, the PMs and solenoids can be a source of error, especially for the extremely low beam emittance required by SuperKEKB. Careful consideration on this point will be required in the future.

This experience of the ECE and its countermeasures in SuperKEKB provide valuable information for designing the vacuum systems of future high-intensity machines $[78,79]$.

\section{ACKNOWLEDGMENTS}

We thank all of the staff of the KEKB accelerator division for their cooperation and continuous encouragement during the commissioning phase.

[1] Y. Ohnishi, Report on SuperKEKB phase 2 commissioning, in Proceedings of the International Particle Accelerator Conference, Vancouver, Canada, 2018 (JACoW, CERN, Geneva, 2018), pp. 1-5.

[2] Y. Ohnishi et al., Accelerator design at SuperKEKB, Prog. Theor. Exp. Phys. (2013) 03A011.

[3] T. Abe et al., Achievements of KEKB, Prog. Theor. Exp. Phys. (2013) 03A001.

[4] K. Ohmi, Beam-Photoelectron Interactions in Positron Storage Rings, Phys. Rev. Lett. 75, 1526 (1995).

[5] K. Ohmi and F. Zimmermann, Head-Tail Instability Caused by Electron Clouds in Positron Storage Rings, Phys. Rev. Lett. 85, 3821 (2000).

[6] F. Zimmermann, The electron cloud instability: Summary of measurements and understanding, in Proceedings of the 2001 Particle Accelerator Conference, Chicago, 2001 (IEEE, New York, 2001), pp. 666-670.

[7] M. T. F. Pivi and M. A. Furman, Electron cloud development in the Proton Storage Ring and in the Spallation Neutron Source, Phys. Rev. ST Accel. Beams 6, 034201 (2003).

[8] G. Rumolo, A. Z. Ghalam, T. Katsouleas, C. K. Huang, V. K. Decyk, C. Ren, W. B. Mori, F. Zimmermann, and F. Ruggiero, Electron cloud effects on beam evolution in a circular accelerator, Phys. Rev. ST Accel. Beams 6, 081002 (2003).

[9] F. Zimmermann, Review of single-bunch instabilities driven by an electron cloud, Phys. Rev. ST Accel. Beams 7, 124801 (2004).

[10] G. Rumolo, H. Bartosik, E. Belli, P. Dijkstal, G. Iadarola, K. Li, L. Mether, A. Romano, M. Schenk, and F.
Zimmermann, Electron cloud effects at the LHC and LHC injectors, in Proceedings of the International Particle Accelerator Conference, Copenhagen, Denmark, 2017 (JACoW, CERN, Geneva, 2017), pp. 30-36.

[11] G. Bregliozzi, V. Baglin, P. Chiggiato, P. Cruikshank, J. M. Jimenez, and G. Lanza, Observations of electron cloud effects with the LHC vacuum system, in Proceedings of the International Particle Accelerator Conference, San Sebastian, Spain, 2011 (JACoW, CERN, Geneva, 2011), pp. 1560-1562.

[12] A. Kulikov, A. Fisher, S. Heifets, J. Seeman, M. Sullivan, and U. Wienands, The electron cloud instability at PEP-II, SLAC Report No. SLAC-PUB-9428, 2002.

[13] G. Rumolo, F. Zimmermann, H. Fukuma, and K. Ohmi, Electron cloud studies for $\mathrm{KEKB}$, in Proceedings of the 2001 Particle Accelerator Conference, 2001 (IEEE, New York, 2001), pp. 1889-1891.

[14] K. Oide, Observations and cures of electron-cloud effects at the KEKB low energy ring, in Proceedings of the Chamonix XI, Chamonix, Switzerland, 2001, CERN Report No. CERN-SL-2001-003, 2001.

[15] M. A. Palmer et al., Electron cloud at low emittance in CESRTA, in Proceedings of the International Particle Accelerator Conference, Kyoto, Japan, 2010 (ICR, Kyoto, 2010), pp. 1251-1255.

[16] F. Zimmermann, Electron-cloud studies for the low energy ring of KEKB, Report No. CERN-SL-Note-2000-004 AP, 2000 .

[17] K. Ohmi and D. Zhou, Study of electron cloud effects in SuperKEKB, in Proceedings of the International Particle Accelerator Conference, Dresden, Germany, 2014 (JACoW, CERN, Geneva, 2014), pp. 1597-1599.

[18] A. Romano, O. Boine-Frankenheim, X. Buffat, G. Iadarola, and G. Rumolo, Electron cloud buildup driving spontaneous vertical instabilities of stored beams in the Large Hadron Collider, Phys. Rev. Accel. Beams 21, 061002 (2018).

[19] F. Zimmermann, H. Fukuma, and K. Ohmi, More electron cloud studies for KEKB: Long-term evolution, solenoid patterns, and fast blowup, CERN Report No. CERN-SLNote-2000-061 AP, 2000.

[20] H. Fukuma et al., Observation of vertical beam blowup in KEKB low energy ring, in Proceedings of the European Particle Accelerator Conference, Vienna, Austria, 2000 (EPS-AG, Vienna, Austria, 2008), pp. 1122-1124.

[21] Y. Susaki and K. Ohmi, Electron cloud instability in SuperKEKB low energy ring, in Proceedings of the 2010 Particle Accelerator Conference, Kyoto, Japan, 2010 (JACoW, CERN, Geneva, 2010), pp. 1545-1547.

[22] Y. Suetsugu, K. Kanazawa, K. Shibata, T. Ishibashi, H. Hisamatsu, M. Shirai, and S. Terui, Results and problems in the construction phase of the SuperKEKB vacuum system, J. Vac. Sci. Technol. A 34, 021605 (2016).

[23] Y. Suetsugu, K. Kanazawa, K. Shibata, T. Ishibashi, H. Hisamatsu, M. Shirai, and S. Terui, Construction status of the SuperKEKB vacuum system, Vacuum 121, 238 (2015).

[24] Y. Suetsugu, K. Kanazawa, K. Shibata, T. Ishibashi, H. Hisamatsu, M. Shirai, and S. Terui, Design and construction of the SuperKEKB vacuum system, J. Vac. Sci. Technol. A 30, 031602 (2012). 
[25] V. Baglin, J. Bojko, O. Gröbner, B. Henrist, N. Hilleret, C. Scheuerlein, and M. Taborelli, The secondary electron yield of technical materials and its variation with surface treatments, in Proceedings of the European Particle Accelerator Conference, Vienna, Austria, 2000 (EPSAG, Vienna, Austria, 2008), pp. 217-219.

[26] B. Henrist, H. Hilleret, C. Scheuerlein, and M. Taborelli, The secondary electron yield of $\mathrm{TiZr}$ and TiZrV nonevaporable getter thin film coatings, Appl. Surf. Sci. 172, 95 (2001).

[27] J. M. Jimenez et al., Electron clouds-Results from SPS and experiments for 2003, in Proceedings of the LHC Performance Workshop-Chamonix XII, Chamonix, Switzerland, 2003, CERN Report No. CERN-AB-2003008 ADM, 2003, pp. 327-334.

[28] V. V. Anashin et al., Experimental investigations of the electron cloud key parameters, CERN Report No. LHC Project Report 313, 1999.

[29] M. Van Gompel, P. Chiggiato, P. Costa Pinto, P. Cruikshank, C. Pasquino, J. Peres Espinos, A. Sapountzis, M. Taborelli, and W. Vollenberg, Amorphous carbon thin film coating of the SPS beam line: Evaluation of the first coating implementation, in Proceedings of the International Particle Accelerator Conference, Copenhagen, Denmark, 2017 (JACoW, CERN, Geneva, 2017), pp. 44-47.

[30] S. Calatroni et al., First accelerator test of vacuum components with laser-engineered surfaces for electroncloud mitigation, Phys. Rev. Accel. Beams 20, 113201 (2017).

[31] C. Yin Vallgren et al., Amorphous carbon coatings for the mitigation of electron cloud in the CERN Super Proton Synchrotron, Phys. Rev. ST Accel. Beams 14, 071001 (2011).

[32] M. A. Palmer et al., The conversion and operation of the Cornell electron storage ring as a test accelerator (CESRTA) for damping rings research and development, in Proceedings of the Particle Accelerator Conference, Vancouver, Canada, 2009 (TRIUMF, Canada, 2010), pp. 4200-4204.

[33] J. R. Calvey et al., Update on electron cloud mitigation studies at CesrTA, in Proceedings of the 2nd International Particle Accelerator Conference, San Sebastián, Spain (EPS-AG, Spain, 2011), pp. 796-798.

[34] J. R. Calvey, W. Hartung, Y. Li, J. A. Livezey, J. Makita, M. A. Palmer, and D. Rubin, Measurements of electron cloud growth and mitigation in dipole, quadrupole, and wiggler magnets, Nucl. Instrum. Methods Phys. Res., Sect. A 770, 141 (2015).

[35] R. E. Kirby and F. K. King, Secondary electron emission yields from PEP-II accelerator materials, Nucl. Instrum. Methods Phys. Res., Sect. A 469, 1 (2001).

[36] F. Le Pimpec, R. E. Kirby, F. King, and M. Pivi, TiN and TiZr-V thin film as a remedy against electron cloud, Nucl. Instrum. Methods Phys. Res., Sect. A 551, 187 (2005).

[37] K. Kennedy, B. Harteneck, G. Millos, M. Benap, F. King, and R. Kirby, TiN coating of the PEP-II low-energy ring aluminum arc vacuum chambers, in Proceedings of the Particle Accelerator Conference, Vancouver, BC, Canada, 1997 (IEEE, New York, 1997), pp. 3568-3570.
[38] D. Alesini, A. Drago, A. Gallo, S. Guiducci, C. Milardi, M. Zobov, S. De Santis, T. Demma, and P. Raimondi, Experimental measurements of e-cloud mitigation using clearing electrodes in the DAФNE collider, in Proceedings of the 3rd International Particle Accelerator Conference, New Orleans, LA, 2012 (IEEE, Piscataway, NJ, 2012), pp. 1107-1109.

[39] D. Alesini, A. Battisti, O. Coiro, T. Demma, S. Guiducci, V. Lollo, C. Milardi, P. Raimondi, M. Serio, R. Sorchetti, and M. Zobov, Design and test of the clearing electrodes for e-cloud mitigation in the e+ DAФNE ring, in Proceedings of the 2010 Particle Accelerator Conference, Kyoto, Japan, 2010 (JACoW, CERN, Geneva, 2010), pp. 1515-1517.

[40] P. He, H. C. Hseuh, M. Mapes, R. Todd, and D. Weiss, Development of titanium nitride coating for SNS ring vacuum chambers, in Proceedings of the 2001 Particle Accelerator Conference, Chicago, 2001 (IEEE, New York, 2001), pp. 2159-2161.

[41] R. Todd, P. He, H. C. Hseuh, and D. Weiss, Summary on titanium nitride coating of SNS ring vacuum chambers, in Proceedings of the 21st Particle Accelerator Conference, Knoxville, TN, 2005 (IEEE, Piscataway, NJ, 2005), pp. 3088-3090.

[42] A. A. Krasnov, Molecular pumping properties of the LHC Arc beam pipe and effective secondary electron emission from cu surface with artificial roughness, Vacuum 73, 195 (2004).

[43] V. V. Anashin, O. B. Malyshev, N. V. Fedorov, V. P. Nazmov, B. G. Goldenberg, I. R. Collins, and O. Gröbner, Reflection of photons and azimuthal distribution of photoelectrons in a cylindrical beam pipe, CERN Report No. LHC Project Report 266, 1999.

[44] Y. Suetsugu et al., R\&D of copper beam duct with antechamber scheme for high current accelerators, Nucl. Instrum. Methods Phys. Res., Sect. A 538, 206 (2005).

[45] Y. Suetsugu, K. Kanazawa, K. Shibata, and H. Hisamatsu, Continuing study on the photoelectron and secondary electron yield of TiN coating and NEG (Ti-Zr-V) coating under intense photon irradiation at the KEKB positron ring, Nucl. Instrum. Methods Phys. Res., Sect. A 556, 399 (2006).

[46] K. Shibata, H. Hisamatsu, K. Kanazawa, M. Shirai, and Y. Suetsugu, Development of TiN coating system for beam ducts of KEK B-factory, in Proceedings of the 11th European Particle Accelerator Conference, Genoa, 2008 (EPS-AG, Genoa, Italy, 2008), pp. 1700-1702.

[47] Y. Suetsugu, H. Fukuma, K. Shibata, M. Pivi, and L. Wang, Beam tests of a clearing electrode for electron cloud mitigation at KEKB positron ring, in Proceedings of the International Particle Accelerator Conference, Kyoto, Japan, 2010 (ICR, Kyoto, 2010), pp. 2369-2371.

[48] Y. Suetsugu, H. Fukuma, M. Pivi, and L. Wang, Continuing study on electron-cloud clearing techniques in high intensity positron ring: Mitigation by using groove surface in vertical magnetic field, Nucl. Instrum. Methods Phys. Res., Sect. A 604, 449 (2009).

[49] Y. Suetsugu, H. Fukuma, K. Shibata, M. Pivi, and L. Wang, Experimental studies on grooved surfaces to suppress secondary electron emission, in Proceedings of the 
International Particle Accelerator Conference, Kyoto, Japan, 2010 (ICR, Kyoto, 2010), pp. 2021-2023.

[50] H. Fukuma, J. Flanagan, K. Hosoyama, T. Ieiri, T. Kawamoto, T. Kubo, M. Suetake, S. Uno, S. S. Win, and M. Yoshioka, Status of solenoid system to suppress the electron cloud effects at the KEKB, in High Intensity and High Brightness Hadron Beams, AIP Conference Proceedings No. 642 (AIP, Geneva, 2002), pp. 357-359.

[51] Y. Funakoshi et al., Beam commissioning of SuperKEKB, in Proceedings of the International Particle Accelerator Conference, Busan, Korea, 2016 (JACoW, CERN, Geneva, 2016), pp. 1019-1021.

[52] J. A. Crittenden, D. C. Sagan, T. Ishibashi, and Y. Suetsugu, Synchrotron radiation analysis of the SuperKEKB positron ring, in Proceedings of the International Particle Accelerator Conference, Richmond, VA, 2015 (JACoW, CERN, Geneva, 2015), pp. 2222-2224.

[53] D. Hunt, K. Kennedy, and T. Stevens, Design of the PEP-II low energy ring vacuum system, in Proceedings of the Particle Accelerator Conference and International Conference on High-Energy Accelerators, Dallas, TX, 1995 (IEEE, Piscataway, NJ, 1996), pp. 2067-2068.

[54] L. F. Wang, D. Raparia, J. Wei, and S. Y. Zhang, Mechanism of electron cloud clearing in the accumulator ring of the Spallation Neutron Source, Phys. Rev. ST Accel. Beams 7, 034401 (2004).

[55] L. Wang, H. Fukuma, K. Ohmi, S. Kurokawa, K. Oide, and F. Zimmermann, Numerical study of the photoelectrons cloud in KEKB low energy ring with a three-dimensional particle in cell method, Phys. Rev. ST Accel. Beams 5, 124402 (2002).

[56] Y. Cai, M. Pivi, and M. A. Furman, Buildup of electron cloud in the PEP-II particle accelerator in the presence of a solenoid field and with different bunch pattern, Phys. Rev. ST Accel. Beams 7, 024402 (2004).

[57] Y. Funakoshi et al., Recent progress at KEKB, in Proceedings of the 10th European Particle Accelerator Conference, Edinburgh, Scotland, 2006 (EPS-AG, Edinburgh, Scotland, 2006), pp. 610-612.

[58] H. Fukuma, J. W. Flanagan, T. Kawamoto, T. Morimoto, K. Oide, M. Tobiyama, and F. Zimmermann, The effect of the solenoid field in quadrupole magnets on the electron cloud instability in the KEKB LER, in Proceedings of the 10th European Particle Accelerator Conference, Edinburgh, Scotland, 2006 (EPS-AG, Edinburgh, Scotland, 2006), pp. 2901-2902.

[59] K. Kanazawa, H. Fukuma, H. Hisamatsu, and Y. Suetsugu, Measurement of the electron cloud density around the beam, in Proceedings of the 21st Particle Accelerator Conference, Knoxville, TN, 2005 (IEEE, Piscataway, NJ, 2005), pp. 1054-1056.

[60] Y. Suetsugu, K. Shibata, T. Ishibashi, K. Kanazawa, M. Shirai, S. Terui, and H. Hisamatsu, First commissioning of the SuperKEKB vacuum system, Phys. Rev. Accel. Beams 19, 121001 (2016).

[61] Y. Suetsugu, K. Shibata, T. Ishibashi, H. Fukuma, M. Tobiyama, J. Flanagan, E. Mulyane, M. Shirai, S. Terui, K. Kanazawa, and H. Hisamatsu, Achievements and problems in the first commissioning of SuperKEKB vacuum system, J. Vac. Sci. Technol. A 35, 03 E103 (2017).
[62] O. Gröbner, A. G. Mathewson, H. Störi, and P. Strubin, Studies of photon induced gas desorption using synchrotron radiation, Vacuum 33, 397 (1983).

[63] O. Gröbner, Beam induced multipactoring, in Proceedings of the Particle Accelerator Conference, Vancouver, Canada, 1997 (TRIUMF, Canada, 1997), pp. 3589-3591.

[64] Y. Suetsugu, Observation and simulation of the nonlinear dependence of vacuum pressures on the positron beam current at the KEKB, in Proceedings of the 2001 Particle Accelerator Conference, Chicago, U.S.A., 2001 (IEEE, New York, 2001), pp. 2183-2185.

[65] M. Tobiyama, J. W. Flanagan, H. Fukuma, S. Kurokawa, K. Ohmi, and S. S. Win, Coupled bunch instability caused by electron cloud, in Proceedings of the 21st Particle Accelerator Conference, Knoxville, TN, 2005 (IEEE, Piscataway, NJ, 2005), pp. 943-945.

[66] S. S. Win, H. Fukuma, K. Ohmi, and S. Kurokawa, Study of coupled bunch instability caused by electron cloud in KEKB positron ring, in Proceedings of the 8th European Particle Accelerator Conference, Paris, France, 2002 (EPAC, France, 2002), pp. 1592-1594.

[67] S. S. Win, K. Ohmi, H. Fukuma, M. Tobiyama, J. Flanagan, and S. Kurokawa, Numerical study of coupled-bunch instability caused by an electron cloud, Phys. Rev. ST Accel. Beams 8, 094401 (2005).

[68] K. Ohmi, J. Flanagan, H. Fukuma, H. Ikeda, E. Mulyani, K. Shibata, Y. Suetsugu, and M. Tobiyama, Electron cloud studies in SuperKEKB phase I commissioning, in Proceedings of the International Particle Accelerator Conference, Copenhagen, Denmark, 2017 (JACoW, CERN, Geneva, 2017), pp. 3104-3106.

[69] S. A. Antipov, P. Adamson, A. Burov, S. Nagaitsev, and M.-J. Yang, Fast instability caused by electron cloud in combined function magnets, Phys. Rev. Accel. Beams 20, 044401 (2017).

[70] Y. Suetsugu, K. Shibata, T. Ishibashi, K. Kanazawa, M. Shirai, S. Terui, and H. Hisamatsu, Beam scrubbing of beam pipes during the first commissioning of SuperKEKB, Appl. Surf. Sci. 432, 347 (2018).

[71] R. Cimino, L. A. Gonzalez, R. Larciprete, A. Di Gaspare, G. Iadarola, and G. Rumolo, Detailed investigation of the low energy secondary electron yield of technical $\mathrm{Cu}$ and its relevance for the LHC, Phys. Rev. ST Accel. Beams 18, 051002 (2015).

[72] R. T. Poole, R. C. G. Leckey, J. G. Jenkin, and J. Liesegang, Photoelectron angular distributions from gold, J. Electron Spectrosc. Relat. Phenom. 1, 371 (1972).

[73] Z. Pei and C. N. Berglund, Angular distribution of photoemission from gold thin films, Jpn. J. Appl. Phys. 41, L52 (2002).

[74] Y. Suetsugu, Y. Tanimoto, Y. Hori, K. Kanazawa, Y-J. Hsu, and M. Kobayashi, Effects of external magnetic fields on the photoelectron emission from a copper beam chamber, in Proceedings of the 2001 Particle Accelerator Conference, Chicago, 2001 (IEEE, New York, 2001), pp. 2180-2182.

[75] G. Iadarola and G. Rumolo, PyECLOUD and build-up simulations at CERN, in Proceedings of the joint INFNCERN-EuCARD-AccNet workshop on electron-cloud 
effects: ECLOUD'12, Isola d'Elba, Italy, 2012, CERN Yellow Report No. CERN-2013-002, pp. 189-194.

[76] G. Dugan and D. Sagan, Synrad3D photon propagation and scattering simulations, in Proceedings of the joint INFN-CERN-EuCARD-AccNet workshop on electroncloud effects: ECLOUD'12, Isola d'Elba, Italy, 2012, CERN Yellow Report No. CERN-2013-002, pp. 117-129.

[77] G. Guillermo, G. H. I. Maury Cuna, E. D. Ocampo Ortiz, and F. Zimmermann, Electron cloud buildup for LHC 'Sawtooth' vacuum chamber, in Proceedings of the International Particle Accelerator Conference, Vancouver
BC, Canada, 2018 (JACoW, CERN, Geneva, 2018), pp. 744-746.

[78] M. T. F. Pivi et al., Recommendation for the feasibility of a $3 \mathrm{~km}$ damping ring, in Proceedings of the International Particle Accelerator Conference, Kyoto, Japan, 2010 (ICR, Kyoto, 2010), pp. 3578-3580.

[79] E. Belli, P. Costa Pinto, G. Rumolo, T. Sinkovits, M. Taborelli, and M. Migliorati, Electron cloud studies in FCC-ee, in Proceedings of the International Particle Accelerator Conference, Vancouver BC, Canada, 2018 (JACoW, CERN, Geneva, 2018), pp. 374-377. 